\title{
Charged rotating black holes in Einstein-Maxwell-Chern-Simons theory with a negative cosmological constant
}

\author{
Jose Luis Blázquez-Salcedo ${ }^{1}$, Jutta Kunz ${ }^{1}$, \\ Francisco Navarro-Lérida ${ }^{2}$, Eugen Radu ${ }^{3}$ \\ ${ }^{1}$ Institut für Physik, Universität Oldenburg \\ Postfach 2503, D-26111 Oldenburg, Germany \\ ${ }^{2}$ Dept. de Física Atómica, Molecular y Nuclear, Ciencias Físicas \\ Universidad Complutense de Madrid, E-28040 Madrid, Spain \\ ${ }^{3}$ Departamento de Física da Universidade de Aveiro and CIDMA, \\ Campus de Santiago, 3810-183 Aveiro, Portugal
}

March 23, 2017

\begin{abstract}
We consider rotating black hole solutions in five-dimensional Einstein-Maxwell-Chern-Simons theory with a negative cosmological constant and a generic value of the Chern-Simons coupling constant $\lambda$. Using both analytical and numerical techniques, we focus on cohomogeneity-1 configurations, with two equal-magnitude angular momenta, which approach at infinity a globally AdS background. We find that the generic solutions share a number of basic properties with the known Cvetič, Lü and Pope black holes which have $\lambda=1$. New features occur as well; for example, when the Chern-Simons coupling constant exceeds a critical value, the solutions are no longer uniquely determined by their global charges. Moreover, the black holes possess radial excitations which can be labelled by the node number of the magnetic gauge potential function. Solutions with small values of $\lambda$ possess other distinct features. For instance, the extremal black holes there form two disconnected branches, while not all near-horizon solutions are associated with global solutions.
\end{abstract}

\section{Introduction}

The study of black hole (BH) solutions with a cosmological constant $\Lambda<0$ has enjoyed recently a tremendous amount of interest. The natural ground state here is the Anti-de Sitter (AdS) spacetime, which, from a mathematical viewpoint, can be regarded as fundamental as the Minkowski one, possessing the same number of Killing vectors [1]. Therefore, finding less symmetric solutions and contrasting the situation with the Minkowskian counterparts is an interesting problem in itself which ultimately may lead to a better understanding of the real world BHs. However, the main motivation for the study of BHs with AdS asymptotics comes from the proposed correspondence between physical effects associated with gravitating fields propagating in AdS spacetime and those of a conformal field theory (CFT) on the boundary of AdS spacetime [2, 3]. According to this conjecture, the $\mathrm{AdS}_{D} \mathrm{BHs}$ would offer the possibility of understanding the nonperturbative structure of some CFTs in $(D-1)$-dimensions.

Restricting to a globally AdS background and $D=5$ spacetime dimensions, one remarks that the asymptotically flat Myers-Perry BH [4] possesses a generalization which has been studied by various authors, starting with the work by Hawking et.al. [5. However, the situation is more patchy in the presence of an (Abelian) gauge field. The only Einstein-Maxwell solution which is known in closed form is the (electrically 
charged, spherically symmetric) Reissner-Nordström-AdS (RN-AdS) BH. The basic properties of its rotating generalization has been studied in [6] by using numerical methods, for the particular case of two equal angular momenta $\left|J_{1}\right|=\left|J_{2}\right|$, while the general solutions with $J_{1} \neq J_{2}$ are still unknown.

However, in five spacetime dimensions the Maxwell action may be supplemented by a Chern-Simons (CS) term. This makes no difference to static configurations but it does affect the class of stationary solutions. Rather than being merely an extension of the Einstein-Maxwell model, the inclusion of a CS term is motivated by its presence (with a particular coefficient $\lambda=\lambda_{S G}=1$ ) in the bosonic sector of $D=5$ minimal gauged supergravity. Several exact solutions of this supergravity model describing charged rotating BH solutions have been reported in the literature. Of main interest here are the $\mathrm{BH}$ solutions found in [7] by Cvetič, Lü and Pope (CLP). These are the most general asymptotically AdS BHs which rotate in two planes with equal-magnitude angular momenta and are free of pathologies. As such, they possess three global charges: the mass $M$, the electric charge $Q$ and the angular momenta $\left|J_{1}\right|=\left|J_{2}\right|=J$. The parameters $M, Q, J$ are subject to some constraints, such that closed timelike curves and naked singularities are avoided. The BHs in [7] also possess an extremal limit which preserves some amount of supersymmetry [8] (note that these solutions present non-vanishing angular momentum). Generalizations of these configurations with more matter fields and/or unequal angular momenta have been constructed in [9].

The main purpose of this paper is to answer the question on how general is the CLP solution? For example, when taking a value $\lambda \neq \lambda_{S G}$ of the CS coupling constant and imposing the following assumptions: i) $\mathrm{AdS}_{5}$ asymptotics; ii) two equal angular momenta; and iii) the absence of pathologies, do we recover the same qualitative features as in [7]?

Although a CS term does not contribute to the Einstein equations, its presence breaks the charge reversal invariance. Moreover, a value $\lambda \neq 0$ introduces a nonlinearity at the level of the Maxwell-Chern-Simons equations; thus varying $\lambda$ may lead to new features of the solutions. Indeed, as discussed in [10, 11], 12, the asymptotically flat limit of these BHs possesses a variety of new properties when the Chern-Simons coupling constant is large enough [starting above the supergravity (SUGRA) value]. Perhaps the most unusual feature there is that the BHs form sequences of radially excited solutions, that can be labeled by the node number of the magnetic gauge potential function. Moreover, the solutions there exhibit non-uniqueness and one finds extremal and non-extremal BHs with the same sets of global charges and different bulk geometries.

It is likely that some of the new features in [10, [11, 12, will survive in the presence of a negative cosmological constant. Thus one can predict that the CLP solution (which possesses a nodeless magnetic gauge potential function) will fail to provide an accurate qualitative description for large enough $\lambda$. However, the situation is less clear for small enough values of the CS coupling constant.

To answer the questions above, we consider the same framework as in the CLP case (in particular the same boundary conditions at infinity) and study Einstein-Maxwell-Chern-Simons (EMCS) BHs with $\lambda \neq \lambda_{S G}$, a task which, to our knowledge, has not been yet undertaken in the literature. Our results show that new qualitative features occur both for small and for large enough values of the CS coupling constant. Then one cannot safely extrapolate the features of the CLP solutions to the case of a generic $\lambda$. In particular, all unusual features found in [10, 11, [12, for asymptotically flat BHs in EMCS theory survive in the presence of a negative cosmological constant. Moreover, new features occur as well for a small $\lambda$.

The paper is organized as follows: in Section 2 we introduce the general framework. The squashed $A d S_{2} \times S^{3}$ solutions of the EMCS model are discussed in Section 3 in conjunction with the near-horizon formalism. Such configurations are of interest since in principle they could emerge as near-horizon limit of the extremal global solutions. The BH solutions are discussed in Sections 4 and 5. Several values of $\lambda$ are considered there, the situation being contrasted with the $\lambda=1$ CLP case. We end with a brief conclusion and outlook in Section 6. The Appendix A contains a discussion of the basic properties of the exact CLP solution. 


\section{$2 \quad$ Framework}

The action for $D=5$ Einstein-Maxwell theory with negative cosmological constant $\Lambda=-6 / L^{2}$ and a Chern-Simons (CS) term is given by

$$
I=-\frac{1}{16 \pi G_{5}} \int_{\mathcal{M}} d^{5} x\left[\sqrt{-g}\left(R+\frac{12}{L^{2}}-F_{\mu \nu} F^{\mu \nu}\right)+\frac{2 \lambda}{3 \sqrt{3}} \varepsilon^{\mu \nu \alpha \beta \gamma} A_{\mu} F_{\nu \alpha} F_{\beta \gamma}\right],
$$

where $R$ is the curvature scalar and $G_{5}$ is Newton's constant in five dimensions; in the following, to simplify the relations, we consider units such that $G_{5}=1$. Also, $A_{\mu}$ is the gauge potential with the field strength tensor $F_{\mu \nu}=\partial_{\mu} A_{\nu}-\partial_{\nu} A_{\mu}$ and $\lambda$ is the CS coupling constant. For the value $\lambda=\lambda_{\mathrm{SG}} \equiv 1$, the action (1) coincides with the bosonic part of $D=5$ minimal gauged supergravity.

The field equations of this model consist of the Einstein equations

$$
G_{\mu \nu}+\Lambda g_{\mu \nu}=2\left(F_{\mu \rho} F_{\nu}^{\rho}-\frac{1}{4} F_{\rho \sigma} F^{\rho \sigma}\right)
$$

together with the Maxwell-Chern-Simons equations

$$
\nabla_{\nu} F^{\mu \nu}+\frac{\lambda}{2 \sqrt{3}} \varepsilon^{\mu \nu \alpha \beta \gamma} F_{\nu \alpha} F_{\beta \gamma}=0 .
$$

The general EMCS rotating BHs would possess two independent angular momenta and a topology of the event horizon which is not necessarily spherical ${ }^{1}$ Thus a generic Ansatz would contain metric functions and gauge potentials with a nontrivial dependence on more than one coordinate. However, this is a very hard numerical problem which we have not yet solved.

The problem is greatly simplified by assuming that the solutions have two equal-magnitude angular momenta and an event horizon with spherical topology. This factorizes the angular dependence of the problem, leading to a cohomogeneity-1 Ansatz, the resulting equations of motion forming a set of coupled nonlinear ODEs in terms of the radial coordinate only ${ }^{2}$. For such solutions the isometry group of the line element is enhanced from $R_{t} \times U(1)^{2}$ to $R_{t} \times U(2)$, where $R_{t}$ denotes the time translation.

An Ansatz with these symmetries is built in terms of the left-invariant 1-forms $\sigma_{i}$ on $S^{3}$, with a line element

$$
d s^{2}=F_{1}(r) d r^{2}+\frac{1}{4} F_{2}(r)\left(\sigma_{1}^{2}+\sigma_{2}^{2}\right)+\frac{1}{4} F_{3}(r)\left(\sigma_{3}-2 W(r) d t\right)^{2}-F_{0}(r) d t^{2},
$$

and a gauge field

$$
A=a_{0}(r) d t+a_{\varphi}(r) \frac{1}{2} \sigma_{3}
$$

where $\sigma_{1}=\cos \psi d \bar{\theta}+\sin \psi \sin \bar{\theta} d \phi, \sigma_{2}=-\sin \psi d \bar{\theta}+\cos \psi \sin \bar{\theta} d \phi, \sigma_{3}=d \psi+\cos \bar{\theta} d \phi$, and $\bar{\theta}, \phi$ and $\psi$ are the Euler angles on $S^{3}$.

\section{Squashed $A d S_{2} \times S^{3}$ solutions and the attractor mechanism}

Some analytical expressions together with a partial understanding of the properties of extremal EMCS BHs can be achieved by considering solutions with a squashed $A d S_{2} \times S^{3}$ geometry. In this case, the $r$-dependence of the problem factorizes such that the field equations reduce to a set of algebraic relations.

\footnotetext{
${ }^{1}$ Vacuum BHs with an $S^{2} \times S^{1}$ event horizon topology in a global $\mathrm{AdS}_{5}$ have been constructed recently in [13]. One expects these solutions to possess generalizations in EMCS theory.

${ }^{2}$ Note that a similar approach has been used by various authors to numerically construct $D=5$ spinning BHs, for various models where an exact solution is missing, see e.g. [14, [15], 16], 17, [18, [19, 20], 21] (perturbative exact solutions have been constructed as well within the same approach, see e.g. 222, 23] 24], 25]).
} 
Such solutions are found by taking a particular expression of the general Ansatz (4) and (5) with

$$
F_{1}=\frac{v_{1}}{r^{2}}, \quad F_{0}=v_{1} r^{2}, \quad F_{2}=v_{2}, \quad F_{3}=v_{2} \eta, \quad W=\alpha r, \quad a_{0}=-q r, \quad a_{\varphi}=p .
$$

The resulting geometry describes a fibration of $A d S_{2}$ over the homogeneously squashed $S^{3}$ with symmetry group $S O(2,1) \times S U(2) \times U(1)\left[26\right.$. In the above relations, $v_{1}, v_{2}, \eta, \alpha, q$ and $p$ are six constants subject to four constraints which result from the EMCS equations (2), (3):

$$
\begin{aligned}
& v_{1}=\frac{L^{2} v_{2}}{4\left(L^{2}+3 v_{2}\right)}, \quad \eta=\frac{L^{4}\left(2 p^{2}-v_{2}\right)}{v_{2}\left(L^{2}+3 v_{2}\right)^{2}-L^{4}}, \\
& q=\frac{L}{4 \sqrt{3}}\left(2-\frac{L^{2}\left(L^{2}+6 p^{2}\right)}{\left(L^{2}+3 v_{2}\right)^{2}}-\frac{L^{2}}{L^{2}+3 v_{2}}-\frac{18 \alpha^{2} L^{2}\left(2 p^{2}-v_{2}\right)}{\left.2 \alpha^{2}\left(L^{2}+3 v_{2}\right)^{2}-L^{4}\right)}\right)^{1 / 2},
\end{aligned}
$$

together with

$$
p=\frac{\alpha \sqrt{\eta} q v_{2}^{2}}{4 v_{1}\left(\sqrt{\eta} v_{1}+\frac{2}{\sqrt{3}} \lambda q \sqrt{v_{2}}\right)} .
$$

A simple solution of the above equations exists in two limiting cases. First, in the absence of a gauge potential $(p=q=0)$ one finds

$$
\eta=2\left(1+\frac{v_{2}}{L^{2}}\right), \quad v_{1}=\frac{v_{2}}{4\left(1+\frac{3 v_{2}}{L^{2}}\right)}, \quad \alpha=\frac{1}{2\left(1+\frac{3 v_{2}}{L^{2}}\right)} \sqrt{\frac{1+\frac{2 v_{2}}{L^{2}}}{1+\frac{v_{2}}{L^{2}}}} .
$$

This solution describes the near-horizon geometry of the extremal Myers-Perry-AdS (MP-AdS) BHs.

For $q \neq 0$, a simple analytic solution can be written for $\lambda=0$ only (i.e. pure EM theory), with

$$
\begin{aligned}
& v_{1}=\frac{L^{2} v_{2}}{4\left(L^{2}+3 v_{2}\right)}, \quad \eta=\frac{4\left(6 v_{2}+4 L^{2}\right) v_{1}^{2}}{\left.L^{2}\left(4 v_{1}^{2}+\alpha^{2} v_{2}^{2}\right)\right)}-\frac{16\left(L^{2}+3 v_{2}\right)^{2}}{L^{4} v_{2}} q^{2} \\
& q=\frac{\sqrt{3 v_{2}}}{4\left(L^{2}+3 v_{2}\right)} \frac{\sqrt{4 \alpha^{2}\left(3 v_{2}^{2}\left(5 L^{2}+v_{2}\right)+L^{4}\left(L^{2}+7 v_{2}\right)\right)-L^{4}\left(L^{2}+2 v_{2}\right)}}{16 \alpha^{4}\left(L^{2}+3 v_{2}^{2}\right)^{4}-L^{8}} .
\end{aligned}
$$

For $\lambda \neq 0$, writing a solution similar to 10 reduces to solving a 6th-order algebraic equation, a task which is approached numerically. Thus we are left with a two-parameter family of solutions, which we found convenient to parametrize in terms of $v_{2}$ and $\eta$. These constants measure the radius of the round $S^{2}$ and the squashing of the $S^{3}$ part of the metric, respectively.

\subsection{Charges}

The solutions of the algebraic system (7)-(8) are expected to describe the near-horizon limit of asymptotically $\mathrm{AdS}_{5}$ extremal BHs in EMCS theory. Thus they are of particular interest in conjunction with the entropy function formalism [27, 28, 29]. For example, this formalism allows us to find the expression for some quantities of interest for the global extremal solutions without integrating the field equations in the bulk; it also leads to some predictions for the structure of those BHs.

The analysis is standard and a detailed computation has been given in [11, for the same framework, although in the absence of a cosmological constant. Thus we shall present here the basic steps only. Following the usual approach, we consider the action functional of the model (with $\mathcal{L}$ the EMCS Lagrangian)

$h\left(\alpha, v_{1}, v_{2}, \eta, p, q\right)=\int d \bar{\theta} d \varphi d \psi \sqrt{-g} \mathcal{L}=4 \pi^{2}\left(\sqrt{\eta v_{2}} v_{1}\left(4-\eta+\frac{6 v_{2}}{L^{2}}+\frac{\alpha^{2} \eta v_{2}^{2}}{4 v_{1}^{2}}\right)+v_{1} \sqrt{\frac{\eta}{v_{2}}}\left(\frac{v_{2}^{2} q^{2}}{v_{1}^{2}}-4 p^{2}\right)-\frac{16 \lambda p^{2} q}{2 \sqrt{3}}\right)$.

The entropy function is the Legendre transform of the above integral with respect to the parameters $\alpha, q$

$$
S=2 \pi(2 \alpha \tilde{J}+\rho \tilde{q}-h),
$$


where $\tilde{J}$ and $\tilde{q}$ are related to the angular momentum and the electric charge of the solutions, respectively.

Within this approach, the Einstein equations correspond to

$$
\frac{\partial h}{\partial v_{1}}=0, \quad \frac{\partial h}{\partial v_{2}}=0, \quad \frac{\partial h}{\partial \eta}=0 .
$$

Note that in the presence of the CS term in the action, the corresponding analysis for the gauge potentials is more intricate [30, and recovering the relations for $(p, q)$ in (7)-(8) requires some care.

Within the entropy function formalism, the considered configurations are characterized by two independent parameters, for which we would like to choose the angular momentum and electric charge. In order to calculate them, one can employ the Noether charges approach [30, 31, 32, 33]. Then the expression of the total angular momentum is 11

$$
J=4 \pi \frac{v_{2}^{3 / 2}}{v_{1}} \sqrt{\eta} p(\rho+p \alpha)+\pi \frac{v_{2}^{5 / 2}}{v_{1}} \eta^{3 / 2} \alpha-\frac{16}{9} \sqrt{3} \pi p^{3} \lambda,
$$

a result which is also obtained from the equation

$$
\frac{\partial h}{\partial \alpha}=\tilde{J} \equiv 16 \pi J
$$

The corresponding expression of the electric charge is [1]

$$
Q=-4 \pi \frac{v_{2}^{3 / 2}}{v_{1}} \sqrt{\eta}(\rho+p \alpha)+\frac{8 \pi \sqrt{3}}{3} \lambda p^{2},
$$

which is equivalent to

$$
\frac{\partial h}{\partial \rho}=\hat{q}=-Q-\frac{8 \pi \sqrt{3}}{9} \lambda p^{2} .
$$

Then the solutions have an entropy

$$
S=\frac{A_{H}}{4},
$$

where

$$
A_{H}=\frac{1}{4} \int d \bar{\theta} d \phi d \psi \sqrt{\left|\operatorname{det}\left(g^{(3)}\right)\right|}=16 \pi^{2} v_{2}^{3 / 2} \sqrt{\eta},
$$

will be identified with the horizon area of the global extremal solutions. For the purposes of this work it is also of interest to consider the horizon angular momentum $J_{H}$, which can be calculated using the standard Komar formula (with the Killing vector $\tau \equiv \partial_{\psi}-\partial_{\phi}$ )

$$
J_{H}=\frac{1}{64 \pi} \int d \bar{\theta} d \phi d \psi \sqrt{-g}\left(\nabla^{r} \tau^{t}-\nabla^{t} \tau^{r}\right)=\pi \frac{v_{2}^{5 / 2}}{v_{1}} \eta^{3 / 2} \alpha .
$$

Note that although the cosmological constant is not explicitly found in the above expressions, it enters via the relations (7)-(8).

\subsection{Branch structure and predictions for the global solutions}

If we fix the CS constant $\lambda$ and the AdS length scale $L$, the near-horizon solutions only depend on the angular momentum $J$ and the electric charge $Q$. Unfortunately, the relations (13), (15), together with (7), (8) are very complicated and thus it is not possible to give an explicit expression for the entropy $S$ as a function of $Q$ and $J$ (the same holds for the horizon angular momentum $J_{H}$ ).

However, it is a straightforward problem to compute those expressions numerically. This reveals a rather complicated picture, with the possible existence of several branches of solutions. 


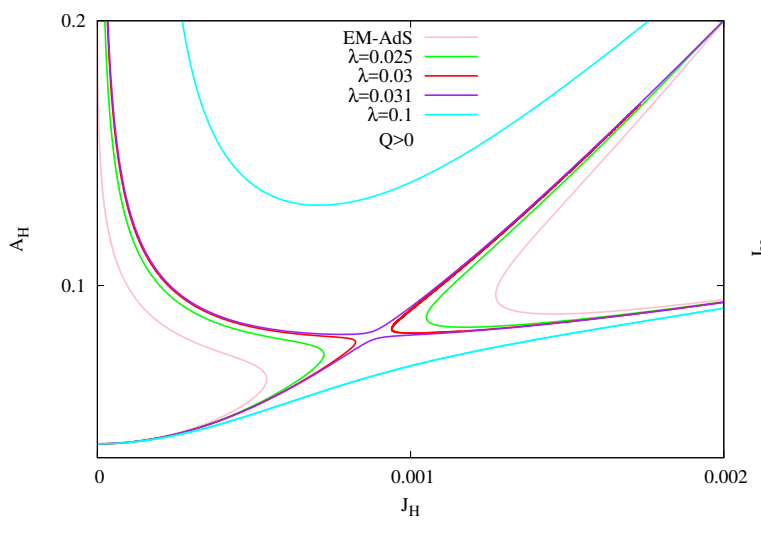

(a) $Q>0$

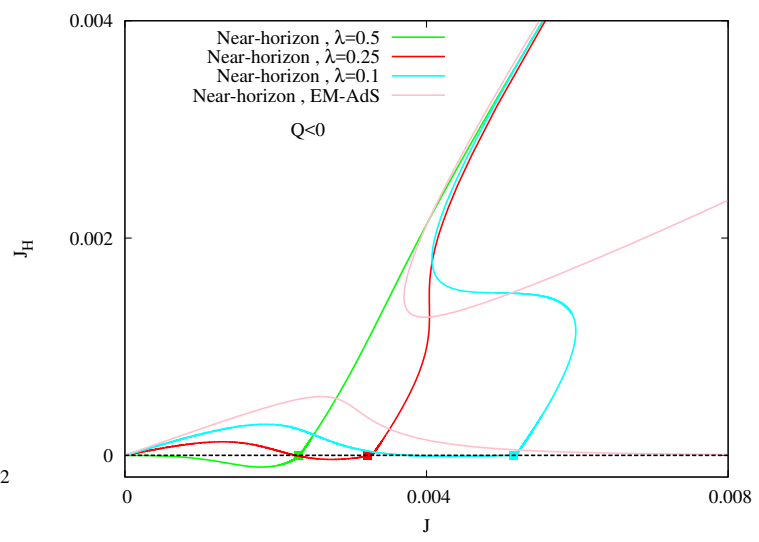

(b) $Q<0$

Figure 1: (a) Area vs. horizon angular momentum $J_{H}$ for near-horizon solutions with fixed positive electric charge $Q=0.044$ and several values of $\lambda$. (b) Horizon angular momentum $J_{H} v s$. angular momentum $J$ for near-horizon solutions with negative electric charge $Q=-0.044$. The squares mark the critical solutions. In both figures we include pure EM near-horizon solutions for reference. Also, the AdS length scale is $L=1$.

\subsection{1 $\lambda=0$ case}

Let us start by addressing first the $\lambda=0$ limit of the solutions, [i.e. Einstein-Maxwell (EM)-AdS theory], in which case partial analytic results are at hand (see the Eqs. (10)). In agreement with our physical intuition, one finds two different branches of solutions, corresponding to two different possible ways to generate extremal EM-AdS global solutions. One of the branches contains the near-horizon geometry of the extremal RN-AdS $\mathrm{BH}$ as the $J=0$ limit. Then a whole branch is generated when adding spin to that limiting solution. Therefore, we call this set the $R N$ branch. The second branch contains the near-horizon geometry of the extremal MP-AdS BH as the uncharged limit. In this case, the whole branch is generated when electric charge is introduced into that near-horizon geometry. As such, this set is called the MP branch.

These two branches are shown in Figure 1 , with a $\left(J_{H}, A_{H}\right)$-diagram (left) and a $\left(J, J_{H}\right)$-diagram (right) (note that the electric charge is fixed there; also all results in this section are found for an AdS length scale $L=1$ ). Interestingly, these two branches never intersect, i.e. there are no attractor solutions connecting them. This leads us to predict that the global solutions will also be on disconnected branches. Thus, for a fixed value of the electric charge, we shall find two branches of extremal BHs, which however, cannot be connected by global solutions whose near-horizon geometry is described by a squashed $A d S_{2} \times S^{3}$ metric.

\subsubsection{Generic picture}

The picture for $\lambda \neq 0$ (i.e. an EMCS-AdS theory) is more complicated. First, in this case, if we fix the CS coupling $\lambda$, the sign of the electric charge becomes relevant. In the following we will assume $\lambda>0$ without any loss of generality and show results for several small values of $\lambda$. For the discussion we shall fix $|Q|=0.044$ and the AdS length to $L=1$, although we have explored other values of these parameters and the features described below are generic.

Let us start with the positive $Q$ case. Then, for the $Q=0.044$ and $0 \leq \lambda<0.0305$, the branch structure is similar to that of the pure EM-AdS case and one finds again two separated branches of near-horizon solutions (see Figure 1ap. One of them, the RN branch, contains the near-horizon limit of the extremal RN-AdS BH. The other one, the MP branch, contains the near-horizon geometry of the extremal MP-AdS BH. Moreover, both branches are disconnected. Then the prediction above for the global solutions still holds for these EMCS solutions.

However, this structure changes drastically for $\lambda \geq 0.0305$, where a bifurcation happens. For this specific value, both branches connect at some particular configuration which does not seem to possess 
special properties. When $\lambda>0.0305$ one finds again two disconnected branches after the bifurcation. These features are shown in Figure 1a, for $\lambda=0.031$ (purple curve) and $\lambda=0.1$ (cyan curve).

Now let us continue with the negative $Q$ case, which presents very different properties. There it is more convenient to show the branch structure by plotting the horizon angular momentum $J_{H} v s$. the total angular momentum $J$, as we do in Figure $1 \mathrm{~b}$. The main feature always present in $Q<0$ solutions is the appearance of a critical near-horizon solution with vanishing horizon area (marked with a square in the Figure). This near-horizon solution is always separating the near-horizon geometry of the extremal MP-AdS BH from the near-horizon geometry of the extremal RN-AdS BH. Hence a prediction of the near-horizon formalism is that in this case we always find (at least) two branches. For fixed $Q$, this critical solution has a certain value of angular momentum $J_{c}$, which increases as $\lambda$ goes to zero (in fact, one finds $J_{c} \rightarrow \infty$ as $\lambda \rightarrow 0$ ). The horizon angular momentum of the critical solutions also vanishes.

For small enough values of $\lambda$, we observe also other interesting features. For instance, consider $0<$ $\lambda<0.25$. Then the MP branch can have more than one solution with the same angular momentum. In Figure 1b, this can be seen for $\lambda=0.1$ (cyan curve), where a vertical line of constant $J$ can intersect up to three times with the curve. However, as $\lambda$ increases this behavior is lost, see the $\lambda=0.25$ curves in Figure 1b. Yet another interesting behavior can be observed on the RN branch. In Figure $1 \mathrm{~b}$ one can see that the near-horizon formalism predicts the existence of counter-rotating solutions. For instance, consider the curves with $\lambda=0.1$ (cyan) and $\lambda=0.25$ (red). It can be seen that for large enough $J$, and for solutions satisfying $J<J_{c}$, the horizon angular momentum goes from positive to negative. In fact for $\lambda>0.5$, the $\mathrm{RN}$ branch always has negative horizon angular momentum, $J_{H}<0$.

We have studied as well solutions with $0.5<\lambda<2$ and it turns out the (qualitative) picture described above for $\lambda=0.5$ holds in that case, in particular the existence of a critical configuration with $A_{H}=0$. A discussion of this property for the $\lambda=\lambda_{S G}$ case is given in Appendix A, based on the analytical CLP solution.

However, new features occur for $\lambda>2$, in which case the branch structure becomes qualitatively similar to that of the asymptotically flat case [11. Essentially, for $Q<0$ the near-horizon geometry of the extremal $\mathrm{RN}-\mathrm{AdS} \mathrm{BH}$ is no longer the only solution with $J=0$, but there is a $J=0$ near-horizon solution, which is not static. We will comment more on that later, when discussing the global aspects of these solutions. The discussion of the branch structure for $\lambda>2$ can be found in [11] (flat case), and, since it is recovered here, we refer the reader to that paper.

\section{Black holes in EMCS-AdS theory. General properties}

\subsection{Parametrization and equations of motion}

To make contact with the previous numerical work on the $D=5 \mathrm{EM}(\mathrm{CS})$ system [6], [1] we introduce the new angular coordinates

$$
\bar{\theta}=2 \theta, \quad \phi=\varphi_{2}-\varphi_{1}, \quad \psi=\varphi_{1}+\varphi_{2},
$$

where $\theta \in[0, \pi / 2], \varphi_{1} \in[0,2 \pi]$ and $\varphi_{2} \in[0,2 \pi]$. Also, we fix the metric gauge and reparametrize the functions in (4) by taking

$$
F_{0}(r)=f(r) N(r), \quad F_{1}(r)=\frac{m(r)}{f(r)} \frac{1}{N(r)}, \quad F_{2}(r)=\frac{m(r)}{f(r)} r^{2}, \quad F_{3}(r)=\frac{n(r)}{f(r)} r^{2}, \quad W(r)=\frac{\omega(r)}{r},
$$

where

$$
N(r)=1+\frac{r^{2}}{L^{2}}
$$


is a 'background' function employed to enforce AdS asymptotics (note that the $\mathrm{AdS}_{5}$ spacetime is recovered for $f=m=n=1, \omega=0$ ). For completeness, we give the corresponding expression of the line element

$$
\begin{aligned}
& d s^{2}=-f(r) N(r) d t^{2}+\frac{m(r)}{f(r)}\left(\frac{d r^{2}}{N(r)}+r^{2} d \theta^{2}\right)+\frac{n(r)}{f(r)} r^{2} \sin ^{2} \theta\left(d \varphi_{1}-\frac{\omega(r)}{r} d t\right)^{2} \\
& +\frac{n(r)}{f(r)} r^{2} \cos ^{2} \theta\left(d \varphi_{2}-\frac{\omega(r)}{r} d t\right)^{2}+\left(\frac{m(r)-n(r)}{f(r)}\right) r^{2} \sin ^{2} \theta \cos ^{2} \theta\left(d \varphi_{1}-d \varphi_{2}\right)^{2}
\end{aligned}
$$

while the corresponding expression of the gauge potential is

$$
A_{\mu} d x^{\mu}=a_{0}(r) d t+a_{\varphi}(r)\left(\sin ^{2} \theta d \varphi_{1}+\cos ^{2} \theta d \varphi_{2}\right) .
$$

With this Ansatz, the Einstein equations reduce to a set of four second-order ordinary differential equations (ODEs) for the metric functions $f, m, n$ and $\omega$

$$
\begin{aligned}
f^{\prime \prime} & -\frac{2 f}{r^{2}}\left(1-\frac{4}{3 N}\right)+f^{\prime}\left(\frac{5 m^{\prime}}{m}+\frac{2 n^{\prime}}{3 n}-\frac{3 f^{\prime}}{2 f}+\frac{N^{\prime}}{N}+\frac{4}{r}\right)+f\left(\frac{N^{\prime}}{3 N}\left(\frac{m^{\prime}}{2 m}+\frac{n^{\prime}}{n}\right)-\frac{m^{\prime}}{3 m}\left(\frac{n^{\prime}}{n}+\frac{m^{\prime}}{2 m}+\frac{4}{r}\right)\right) \\
- & \frac{2}{3 r}\left(\frac{n^{\prime}}{n}+\frac{n}{r N m}\right)-\frac{7 n\left(\omega-r \omega^{\prime}\right)^{2}}{6 r^{2} f N}+\frac{2 f}{L^{2} N}\left(1-\frac{2 m}{f}+\frac{L^{2} N^{\prime}}{r}\right)-\frac{2 f^{2}}{r^{2}}\left(\frac{4 a_{\varphi}^{2}}{r^{2} N m}+\frac{5\left(r a_{0}^{\prime}+w a_{\varphi}^{\prime}\right)^{2}}{3 f^{2} N}+\frac{a_{\varphi}^{\prime 2}}{3 n}\right)=0, \\
m^{\prime \prime} & +\frac{m f^{\prime}}{r f}\left(1+\frac{r N^{\prime}}{2 N}+\frac{r n^{\prime}}{3 n}\right)+\frac{m^{\prime}}{3}\left(\frac{4}{r}+\frac{f^{\prime}}{2 f}+\frac{5 N^{\prime}}{2 N}-\frac{5 m^{\prime}}{2 m}\right)-\frac{m n^{\prime}}{3 r n}\left(\frac{1}{N}+\frac{r m^{\prime}}{2 m}\right) \\
& -\frac{4 m n\left(\omega-r \omega^{\prime}\right)^{2}}{3 r^{2} f^{2} N}+\frac{8 m}{r^{2} N}\left(\frac{r^{2}}{L^{2}}\left(1-\frac{m}{f}\right)-\frac{1}{3}\left(1-\frac{n}{m}\right)\right)-\frac{8 m}{3 r^{2} f N}\left(\left(r a_{0}+\omega a_{\varphi}^{\prime}\right)^{2}+\frac{N f^{2} a_{\varphi}^{\prime 2}}{2 n}\right)=0, \\
n^{\prime \prime} & +\frac{n f^{\prime}}{r f}\left(1+\frac{m N^{\prime}}{2 N}\right)-\frac{m^{\prime} n}{3 m}\left(\frac{N^{\prime}}{2 N}+\frac{m^{\prime}}{m}+\frac{5}{r}\right)+\frac{n}{3}\left(\frac{2 f^{\prime} m^{\prime}}{f m}-\frac{f^{\prime} n^{\prime}}{2 f n}-\frac{m^{\prime} n^{\prime}}{2 m n}\right)+n^{\prime}\left(\frac{8}{3 r}+\frac{7 N^{\prime}}{6 N}-\frac{n^{\prime}}{2 n}\right) \\
& +\frac{8 n}{r^{2} N}\left(\frac{2}{3}\left(1-\frac{n}{m}\right)+\frac{r^{2}}{L^{2}}\left(1-\frac{m}{f}\right)\right)-\frac{\left.n^{2}\left(\omega-r \omega^{\prime}\right)^{2}\right)}{3 r^{2} f^{2} N}+\frac{8 f}{r^{2} N}\left(\frac{1}{3} N a_{\varphi}^{\prime 2}-\frac{2 n}{r^{2} n} a_{\varphi}^{2}-\frac{n}{3 f^{2}}\left(r a_{0}^{\prime}+\omega a_{\varphi}^{\prime}\right)^{2}\right)=0, \\
w^{\prime \prime} & +\frac{w}{r}\left(-\frac{3}{r}+\frac{5 f^{\prime}}{2 f}-\frac{m^{\prime}}{2 m}-\frac{3 n^{\prime}}{2 n}\right)+w^{\prime}\left(\frac{3}{r}-\frac{5 f^{\prime}}{2 f}+\frac{m^{\prime}}{2 m}+\frac{3 n^{\prime}}{2 n}\right)-\frac{4 f a \varphi^{\prime}}{r n}\left(a_{0}^{\prime}-\frac{w^{\prime} a_{\varphi}}{r}\right)=0,
\end{aligned}
$$

together with the 1st-order constraint equation

$$
\begin{aligned}
& \frac{m^{\prime} n^{\prime}}{m n}-\frac{f^{\prime} n^{\prime}}{f n}-\frac{f^{\prime} m^{\prime}}{f m}-\frac{3 f^{\prime} N^{\prime}}{4 f N}+\left(\frac{4 m^{\prime}}{r m}+\frac{2 n^{\prime}}{r n}-\frac{3 f^{\prime}}{r f}\right)\left(1+\frac{r N^{\prime}}{4 N}\right)+\frac{m^{\prime 2}}{2 m^{2}}+\frac{n\left(\omega-r \omega^{\prime}\right)^{2}}{2 r^{2} f^{2} N} \\
& +\frac{2}{r^{2} N}\left(\frac{n}{m}-\frac{6 r^{2} n}{L^{2} f}\right)-\frac{2\left(L^{2}-6 r^{2}\right)}{r^{2} L^{2} N}+\frac{2}{r^{2} N}\left(\frac{\left(r a_{0}^{\prime}+\omega a_{\varphi}^{\prime}\right)^{2}}{f}-\frac{f N a_{\varphi}^{\prime 2}}{n}+\frac{4 f a_{\varphi}^{2}}{r^{2} n}\right)=0 .
\end{aligned}
$$

The gauge potentials $a_{0}, a_{\varphi}$ satisfy the 2nd-order ODEs

$$
\begin{aligned}
& a_{0}^{\prime \prime}+\frac{w a_{\varphi}^{\prime}}{r^{2}}\left(1-\frac{r N^{\prime}}{N}+\frac{r n^{\prime}}{n}-\frac{2 r f^{\prime}}{f}\right)+\frac{n \omega}{r N}\left(\frac{a_{0}^{\prime}\left(r \omega^{\prime}-\omega\right)}{f^{2}}+\frac{4 a_{\varphi}}{r^{2} m}-\frac{\omega^{2} a_{\varphi}^{\prime}}{r f^{2}}\right) \\
&+a_{0}^{\prime}\left(\frac{3}{r}-\frac{3 f^{\prime}}{2 f}+\frac{m^{\prime}}{2 m}+\frac{n^{\prime}}{2 n}\right)+\frac{a_{\varphi}^{\prime} \omega^{\prime}}{r}\left(1+\frac{n \omega^{2}}{f^{2} N}\right)-\lambda \frac{8 a_{\varphi}}{r^{3} \sqrt{3 f m n}}\left(\frac{n \omega}{N}\left(r a_{0}^{\prime}+\omega a_{\varphi}^{\prime}\right)-f^{2} a_{\varphi}\right)=0, \\
& a_{\varphi}^{\prime \prime}-\frac{4 a_{\varphi} n}{r^{2} N m}+a_{\varphi}^{\prime}\left(\frac{1}{r}+\frac{N^{\prime}}{N}+\frac{f^{\prime}}{2 f}+\frac{m^{\prime}}{2 m}-\frac{n^{\prime}}{2 n}+\frac{n \omega}{r f^{2} N}\left(r \omega^{\prime}-\omega\right)\right) \\
&+\frac{n a_{0}^{\prime}}{f^{2} N}\left(\omega-r \omega^{\prime}\right)+\lambda \frac{8 a_{\varphi}\left(r a_{0}^{\prime}+\omega a_{\varphi}^{\prime}\right)}{r^{2} N \sqrt{\frac{3 f m}{n}}}=0 .
\end{aligned}
$$


The equations for $a_{0}$ and $w$ have a total derivative structure, which implies the existence of the first integrals

$$
\begin{aligned}
& a_{0}^{\prime}+\frac{\omega}{r} a_{\varphi}^{\prime}-\frac{4 \lambda}{\sqrt{3}} \frac{f^{3 / 2} a_{\varphi}^{2}}{r^{3} \sqrt{m n}}=\frac{2 f^{3 / 2}}{\sqrt{m n} r^{3} \pi} Q, \\
& \frac{8 Q}{\pi} a_{\varphi}+\frac{16 \lambda}{3 \sqrt{3}} a_{\varphi}^{3}-\frac{n^{3 / 2} \sqrt{m} r^{3}}{f^{5 / 2}}\left(r \omega^{\prime}-\omega\right)=\frac{16}{\pi} J,
\end{aligned}
$$

with the constants of integration $Q, J$, corresponding to the angular momentum and electric charge of the solutions, respectively (see the relations 290 and $(30)$ ).

\subsection{Far field asymptotics and global charges}

The far field expression of the solutions can be constructed in a systematic way, the first terms of the expansion at infinity being

$$
\begin{aligned}
f(r) & =1+\frac{\alpha}{r^{4}}-\frac{2}{21} \frac{9 L^{2} \pi^{2} \alpha-12 L^{2} Q^{2}-2 \pi^{2} \hat{\mu}^{2}}{\pi^{2} r^{6}}+\ldots \\
m(r) & =1+\frac{\beta}{r^{4}}-\frac{1}{21} \frac{15 L^{2} \pi^{2} \alpha-20 L^{2} Q^{2}-8 \pi^{2} \hat{\mu}^{2}}{\pi^{2} r^{6}}+\ldots \\
n(r) & =1+\frac{3(\alpha-\beta)}{r^{4}}-\frac{5}{21} \frac{3 L^{2} \pi^{2} \alpha-4 L^{2} Q^{2}+4 \pi^{2} \hat{\mu}^{2}}{\pi^{2} r^{6}}+\ldots \\
\omega(r) & =\frac{\hat{J}}{r^{3}}-\frac{4 q}{3} \frac{\hat{\mu}}{r^{5}}+(2 \beta-\alpha) \frac{\hat{J}}{r^{7}}+\ldots \\
a_{\varphi}(r) & =\frac{\hat{\mu}}{r^{2}}+\frac{1}{9} \frac{2 L^{2} \sqrt{3} Q \lambda \hat{\mu}-3 Q L^{2} \hat{J}+3 \pi \alpha \hat{\mu}-6 \pi \beta \hat{\mu}}{\pi r^{6}}+\ldots \\
a_{0}(r) & =-\frac{Q}{\pi r^{2}}-\frac{1}{9} \frac{2 \sqrt{3} \pi \lambda \hat{\mu}^{2}+3 \hat{\mu} \hat{J} \pi+3 Q \beta}{\pi r^{6}}+\ldots
\end{aligned}
$$

The expression of the higher-order terms is rather complicated, and it has not been possible to identify a general pattern for the coefficients. Here we mention only that this asymptotic expansion contains five undetermined parameters $\{\alpha, \beta, \hat{J}, \hat{\mu}, Q\}$, which encode the global charges of the solutions.

The total angular momenta of the $\mathrm{BH}$ can be calculated using the standard Komar integral

$$
J_{(k)}=\int_{S_{\infty}^{3}} \hat{\beta}_{(k)},
$$

where $\hat{\beta}_{(k) \mu_{1} \mu_{2} \mu_{3}} \equiv \epsilon_{\mu_{1} \mu_{2} \mu_{3} \rho \sigma} \nabla^{\rho} \eta_{(k)}^{\sigma}\left(\right.$ with $\left.\eta_{(k)} \equiv \partial / \partial_{\varphi_{(k)}}\right)$. These configurations have equal-magnitude angular momenta, $\left|J_{(k)}\right|=|J|, k=1,2$. Then one finds the following expression

$$
J=\frac{\pi}{4} \hat{J} .
$$

The computation of the electric charge is also standard, $Q$ being obtained from

$$
Q=-\frac{1}{2} \int_{S_{\infty}^{3}}\left(\tilde{F}+\frac{\lambda}{\sqrt{3}} A \wedge F\right)
$$

with $\tilde{F}_{\mu_{1} \mu_{2} \mu_{3}} \equiv \epsilon_{\mu_{1} \mu_{2} \mu_{3} \rho \sigma} F^{\rho \sigma}$.

The computation of the total mass $M$, however, requires special care, since the result from a naive application of the Komar integral diverges already in the vacuum case without the gauge field. However, $M$ can be computed e.g. by using the Ashtekar-Magnon-Das conformal mass definition [34, which results in

$$
M=-\frac{\pi}{8} \frac{(3 \alpha+\beta)}{L^{2}} .
$$


$M$ can also be computed by employing the boundary counterterm approach in [35, wherein it is the conserved charge associated with Killing symmetry $\partial / \partial t$ of the induced boundary metric, found for a large constant value of $r$. This results in the same expression (31), plus a constant Casimir term $M_{0}=\frac{3 \pi}{32} L^{2}$ [35], which we shall ignore in what follows. We mention that $J$ can also be computed by using the approach in [34] or the one in [35, the results coinciding with 29]. Let us also note that $M$ and $J$ are evaluated relative to a frame which is nonrotating at infinity.

The solutions possess also a magnetic moment $\mu_{\text {mag }}$ which is fixed by the constant $\hat{\mu}$ which enters the asymptotic expansion of the gauge potential $a_{\varphi}$,

$$
\mu_{\mathrm{mag}}=\pi \hat{\mu} .
$$

Thus, one can define a gyromagnetic ratio $g$

$$
\mu_{\mathrm{mag}}=g \frac{Q J}{2 M} .
$$

\subsection{Properties of the event horizon}

In the quasi-isotropic coordinates we are employing, the $\mathrm{BH}$ horizon $\mathcal{H}$ resides at $r=r_{H} \geq 0$ (where the function $f$ vanishes), and rotates with angular velocity $\Omega_{H}$. This is a Killing horizon, since the Killing vector

$$
\zeta=\partial_{t}+\Omega_{H}\left(\partial_{\varphi_{1}}+\partial_{\varphi_{2}}\right)
$$

becomes null and orthogonal to the other Killing vectors on it, $\left.\left(\zeta^{2}\right)\right|_{\mathcal{H}}=0,\left.\left(\zeta \cdot \partial_{t}\right)\right|_{\mathcal{H}}=0,\left.\left(\zeta \cdot \partial_{\varphi_{(k)}}\right)\right|_{\mathcal{H}}=0$.

For nonextremal solutions, the following expansion holds near the event horizon:

$$
\begin{aligned}
f(r) & =f_{2}\left(r-r_{H}\right)^{2}-f_{2}\left(\frac{1}{r_{H}}+\frac{3 r_{H}}{L^{2}+r_{H}^{2}}\right)\left(r-r_{H}\right)^{3}+O\left(r-r_{H}\right)^{4}, \\
m(r) & =m_{2}\left(r-r_{H}\right)^{2}-3 m_{2}\left(\frac{1}{r_{H}}+\frac{r_{H}}{L^{2}+r_{H}^{2}}\right)\left(r-r_{H}\right)^{3}+O\left(r-r_{H}\right)^{4}, \\
n(r) & =n_{2}\left(r-r_{H}\right)^{2}-3 n_{2}\left(\frac{1}{r_{H}}+\frac{r_{H}}{L^{2}+r_{H}^{2}}\right)\left(r-r_{H}\right)^{3}+O\left(r-r_{H}\right)^{4}, \\
\omega(r) & =\omega_{0}+\frac{\omega_{0}}{r_{H}}\left(r-r_{H}\right)+O\left(r-r_{H}\right)^{2}, \\
a_{0}(r) & =a_{0}^{(0)}+a_{0}^{(2)}\left(r-r_{H}\right)^{2}+O\left(r-r_{H}\right)^{3}, \\
a_{\varphi}(r) & =a_{\varphi}^{(0)}+a_{\varphi}^{(2)}\left(r-r_{H}\right)^{2}+O\left(r-r_{H}\right)^{3},
\end{aligned}
$$

where $\left\{f_{2}, m_{2}, n_{2}, \omega_{0} ; a_{0}^{(0)}, a_{0,2}, a_{\varphi}^{(0)}, a_{\varphi}^{(2)}\right\}$ are numerical coefficients subject to the constraint

$$
\begin{aligned}
& \left(54 r_{H}^{4}+\frac{71}{2} L^{2} r_{H}^{2}+\frac{29}{4} L^{4}\right) f_{2} m_{2}+10 L^{2}\left(L^{2}+2 r_{H}^{2}\right) f_{2} n_{2}+12 r_{H}^{2}\left(L^{2}+2 r_{H}^{2}\right) m_{2}^{2} \\
& -L^{2} r_{H}^{2}\left(5 L^{2}+13 r_{H}^{2}\right) w_{2} \frac{m_{2} n_{2}}{f_{2}}-8 L^{2} r_{H}^{4}\left(a_{0}^{(2)}+\Omega_{H} a_{\varphi}^{(2)}\right) m_{2}+\frac{4 L^{2}\left(5 L^{2}+9 r_{H}^{2}\right)}{r_{H}^{2}} a_{\varphi}^{(0) 2} f_{2}^{2}=0 .
\end{aligned}
$$

Note that for extremal BHs the event horizon is located at $r_{H}=0$, in which case the near-horizon expansion is more complicated [11. Also, this results in a different expression of the horizon quantities as compared to the one above. by

Restricting to the non-extremal case, the area of the horizon $A_{H}$ and the Hawking temperature are given

$$
A_{H}=\int_{\mathcal{H}} \sqrt{\left|g^{(3)}\right|}=2 \pi^{2} r_{H}^{3} \frac{m_{2}}{f_{2}} \sqrt{\frac{n_{2}}{f_{2}}}, \quad T_{H}=\frac{1}{2 \pi}\left(1+\frac{r_{H}^{2}}{L^{2}}\right) \frac{f_{2}}{\sqrt{m_{2}}} .
$$

The horizon angular velocity is obtained in terms of the inertial dragging

$$
\Omega_{H}=\frac{\omega_{0}}{r_{H}} .
$$


Further, the horizon electrostatic potential $\Phi_{H}$ is defined by

$$
\Phi_{H}=\left.\zeta^{\mu} A_{\mu}\right|_{r=r_{H}}=a_{0}^{(0)}+\Omega_{H} a_{\varphi}^{(0)},
$$

being constant at the horizon.

It is also of interest to compute the horizon mass $M_{H}$ and the horizon angular momenta $J_{H(k)}$, which are given by the standard Komar integrals (with $\hat{\alpha}_{\mu_{1} \mu_{2} \mu_{3}} \equiv \epsilon_{\mu_{1} \mu_{2} \mu_{3} \rho \sigma} \nabla^{\rho} \xi^{\sigma}$ ):

$$
\begin{aligned}
& M_{H}=-\frac{3}{2} \int_{\mathcal{H}} \hat{\alpha}=\frac{3}{16} \pi r_{H}^{3} \sqrt{\frac{m_{2} n_{2}}{f_{2}^{3}}}\left(2 f_{2}\left(1+\frac{r_{H}^{2}}{L^{2}}\right)-\frac{2 r_{H} n_{2} \Omega_{H} w_{2}}{f_{2}}\right), \\
& J_{H(k)}=\int_{\mathcal{H}} \hat{\beta}_{(k)}=-\frac{1}{8} \pi \sqrt{\frac{m_{2} n_{2}^{3}}{f_{2}^{5}}} r_{H}^{4} w_{2} .
\end{aligned}
$$

In the case we are interested here both horizon angular momenta have the same magnitude so we can refer to them simultaneously as the horizon angular momentum $J_{H}$, with $\left|J_{H}\right|=\left|J_{H(1)}\right|=\left|J_{H(2)}\right|$.

Note that the quantities above satisfy the horizon Smarr formula

$$
\frac{2}{3} M_{H}=\frac{\kappa A_{H}}{8 \pi}+2 \Omega_{H} J_{H} .
$$

However, different from the asymptotically flat case, no simple Smarr-type relation can be written for asymptotically AdS configurations, in particular for the solutions in this work. A proposed generalized Smarr-type relation could include the cosmological constant as a negative pressure term [36]. This possibility has been explored for MP-AdS, RN-AdS and CPL BHs in [36, 37.

Finally, we mention that the EMCS charged spinning BHs satisfy the 1st law of thermodynamics

$$
d M=\frac{1}{4} T_{H} d A_{H}+2 \Omega_{H} d J+\Phi_{H} d Q .
$$

An extra term involving variations of the cosmological constant $(\Theta d \Lambda \equiv-V d P)$ can also be added to this formula [36, 37]. The conjugate variable to the pressure $P$ can be identified with the volume $V$ of the space-time outside the event horizon. However in our calculations we will always consider families of configurations with a fixed value of the cosmological constant. The extension of the results in [36], [37] for numerical solutions (in particular for those in this work) remains an interesting open problem.

\section{Black holes in EMCS-AdS theory. Numerical results}

\subsection{General remarks}

\subsubsection{Method}

Unfortunately, no exact EMCS-AdS closed-form solution is known apart from the CLP one (with $\lambda=1$ ) with its RN-AdS $(J=0)$ and MP-AdS $(\mathrm{Q}=0)$ limits. The basic features of this special solution are discussed in Appendix A.

The BHs with $\lambda \neq 1$ are found numerically. The numerical methods we have used are similar to those used in the literature to find numerically other $D=5 \mathrm{BH}$ solutions with equal-magnitude angular momenta, e.g. those in [11, 14, 18, 19. In our scheme, we choose to solve a system of four second-order differential equations (ODEs) for the functions $\left.\left(f, m, n, a_{\varphi}\right)(23), 225\right)$, together with the two first-order ODEs for $\omega, a_{0},(26)$. Thus, in the generic case, the input parameters are $\lambda, L$ together with $r_{H}, J, Q$. The equations are solved by using the software package COLSYS [38, subject to appropriate boundary conditions compatible with the asymptotics (27), (35). This solver employs a collocation method for boundary-value ordinary differential equations and a damped Newton method of quasi-linearization. A linearized problem is solved at each iteration step, by using a spline collocation at Gaussian points. The package COLSYS possesses 


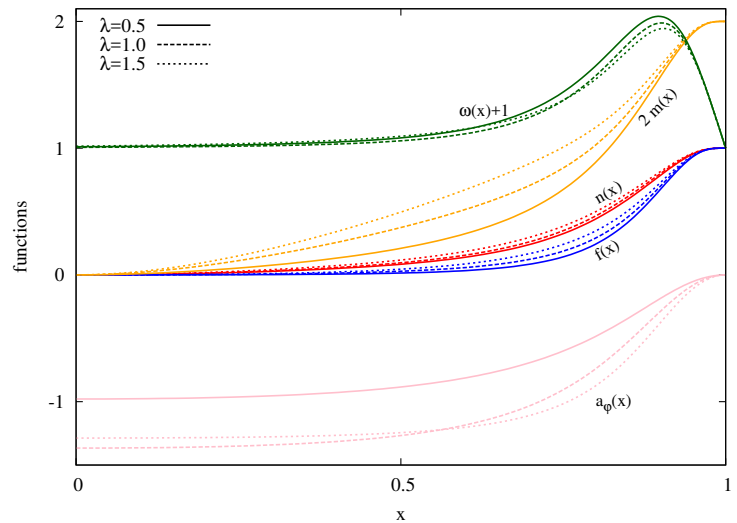

Figure 2: The profiles of typical charged rotating black holes with several values of $\lambda$ and $Q=-0.044$, $J=0.00148, r_{H}=0.4, L=1$ are shown as a function of the compactified coordinate $x=1-r_{H} / r$.

an adaptive mesh selection procedure, such that the equations are solved on a sequence of meshes until the successful stopping criterion is reached.

The solutions reported in this work have a typical relative accuracy of $10^{-10}$. The number of mesh points used in our calculation was around $10^{4}$, distributed non-equidistant on $x$, where $x=1-r_{H} / r$ is a compactified radial coordinate employed in the non-extremal case (for extremal solutions we have used $x=r /(1+r))$.

\subsubsection{The profile of solutions and some generic features}

The profiles of three typical solution $\$ 3$ with $\lambda=0.5, \lambda=\lambda_{S G}=1, \lambda=1.5$ and fixed values $Q=-0.044$, $J=0.00148, r_{H}=0.4, L=1$, are shown in Figure 2. Varying $\lambda$ does not seem to lead to new qualitative features; in particular the metric functions $f, m$ and $n$ always exhibit a monotonic behavior. Also, we have noticed that the difference between solutions' profiles for different $\lambda$ and the same $Q, J, r_{H}$ becomes more transparent when the BHs are close to extremality.

We mention that all solutions reported in this work have $g^{t t}=-f<0$, for any $r>r_{H}$, while $m$ and $n$ remain strictly positive. Thus $t$ is a global time function and the BHs are free of closed timelike (or null) curves [39. Also, they show no sign of a singular behavior ${ }^{4}$ on the horizon or outside of it, that would manifest itself in the Ricci or Kretschmann scalars (which were monitored for most of the solutions). In addition, all the solutions we have analyzed present an ergoregion, inside of which the observers cannot remain stationary and will move in the direction of rotation. The ergoregion is located between the horizon and the ergosurface $r=r_{c}$, with $g_{t t}\left(r_{c}\right)=0$, i.e.

$$
\frac{n\left(r_{c}\right)}{f\left(r_{c}\right)} \omega^{2}\left(r_{c}\right)-f\left(r_{c}\right)\left(1+\frac{r_{c}^{2}}{L^{2}}\right)=0
$$

(note that, in contrast to $D=4$ Kerr-like BHs, the ergosurface does not touch the horizon).

The determination of the full domain of existence of the solutions would be a huge task. In this work, we will only attempt to sketch its shape by analyzing the pattern of solutions on some generic surfaces in the space of parameters. Also, to simplify the study, we set the AdS length scale $L=1$, such that all quantities are given in these units. Moreover, without any loss of generality, we consider values $\lambda \geq 0$ for the CS coupling constant, only (as such, we have to consider both signs for the electric charge). We have considered solutions with a large set of $\lambda$ ranging between 0 and 50. However, solutions with larger $\lambda$ are very likely to exist and we conjecture the absence of an upper bound for the CS coupling constant.

\footnotetext{
${ }^{3}$ As discussed below, for large enough values of $\lambda$, new sets of excited solutions occur, with a node structure for both $a_{\varphi}$ and $\omega$.

${ }^{4}$ The exception here appears to be the gap set of extremal solutions discussed in Subsection 5.3 .
} 
We mention also that the numerical results exhibited in the Figures in Section 5.2 were found by extrapolating to the continuum the results from discrete sets of around one thousand solutions for each $\lambda$. The solutions there were found by considering first a fixed angular momentum (Subsection 5.2.1) and then a fixed electric charge (Subsection 5.2.2). Those plots are (typically) projections of 3D surfaces which encode the dependence of the Hawking temperature $T_{H}$ on two other quantities which enter the 1st law 43.

As such, viewed together, they provide a description of the thermodynamics of the solutions, together with the domain of existence. For example, one can consider the thermodynamic stability in the canonical ensemble, where the charge and angular momentum are fixed parameters, the response function being the heat capacity $C=T_{H}\left(\frac{\partial A_{H}}{\partial T_{H}}\right)_{J, Q}$. We have found that for any value of $\lambda$, the solutions with small values of $|J|,|Q|$ exhibit the pattern of the Schwarzschild-AdS BHs [40], only the large size BHs possessing a positive specific heat $C>0$. However, the solutions become more thermally stable as $|Q|$ and/or $|J|$ increase, with $C>0$ for large enough values of these charges even for small size BHs.

Apart from the quantities displayed in the Figures in Section 5.2, we have also considered the gyromagnetic ratio $g$ as resulting from (33). A known result here is that, unlike in four dimensions, the value of $g$ is not universal in higher dimensions [41, while the AdS asymptotics further introduces new features [42. We have computed the gyromagnetic ratio for a large part of the solutions reported in this work and could not identify any clear pattern, with $g$ taking a large range of values.

Finally, let us mention that in the numerical study we have paid special attention to extremal BHs, which have been constructed directly. Such configurations are important in themselves; they are also interesting as a test of the predictions in Section 3 within the near-horizon formalism.

\subsection{Three values of $\lambda$ : a comparison}

To clarify the question asked in the Introduction on "how general the features of the CLP solution are", we shall present in what follows the results for three intermediate values of the Chern-Simons coupling constant. Apart from the SUGRA case $\lambda=1$, we shall exhibit results for a smaller value, $\lambda=0.5$, and also for a larger one, $\lambda=1.5$.

\subsubsection{A fixed angular momentum: the generic picture}

Starting with extremal BHs with a $Q>0$, one finds one single branch of solutions which connects continuously the extremal MP-AdS BH $(Q=0)$ with the extremal RN-AdS BH (in the limit $Q \rightarrow \infty)$. This fact agrees with the prediction from the near-horizon formalism in Section 3.

For $Q<0$ the situation changes drastically. In agreement with the prediction from the near-horizon formalism, this set is characterized by the existence of two different branches, the MP one and the RN one, separated by a critical solution. The MP branch starts with the extremal MP-AdS solution $(Q=0)$, and extends for $Q \in\left(Q_{0}, 0\right]$, where $Q_{0}<0$. In particular, for $J=0.0295$, one finds $Q_{0}=-0.0522$ for $\lambda=0.5$, $Q_{0}=-0.0659$ in the SUGRA case, and $Q_{0}=-0.0755$ for $\lambda=1.5$. On the other hand, the RN branch exists for $Q \in\left(-\infty, Q_{0}\right)$.

These features are shown in Figure 3 where we give the $\left(A_{H}, Q ; T_{H}\right)$ plot for BHs with $J=0.0295$. One can see that, in all cases, the horizon area is minimized by the set of extremal solutions. One can also notice the different behavior of the $T_{H}=0$ BHs with positive and negative $Q$. For extremal solutions with $Q>0$, the area always increases with the electric charge. However, for $Q<0$, the horizon area becomes zero at the critical solution, which is reached for some critical electric charge $Q_{0}$.

Let us discuss now the non-extremal solutions. In Figure 3 we can see that all three cases possess a local maximum of the temperature. This local maximum is at $T_{H}=0.68$ and is always found for the $Q=0$ MP-AdS set. However, for higher values of the horizon area, the temperature increases again (not displayed in these Figures). Also, there is no upper bound for the area. Another interesting feature that one can see in Figure 3 is that it is possible to define closed sets of charged BHs with the same temperature (isothermal). These sets can only be found around the local maximum of temperature.

From these Figures we conclude that the general behavior of the area does not change much with respect to the SUGRA solution. The effect of changing $\lambda$ reduces to a modification of the position of the critical 


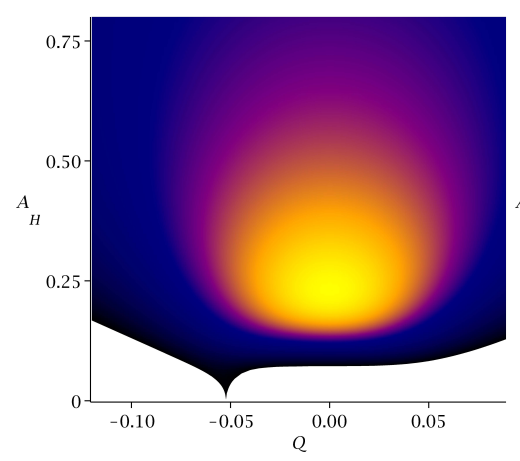

(a) $\lambda=0.5$

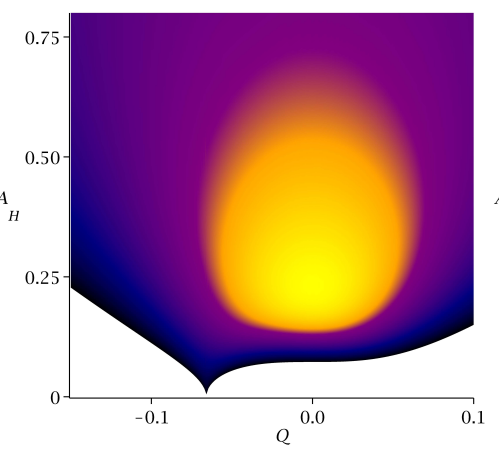

(b) SUGRA

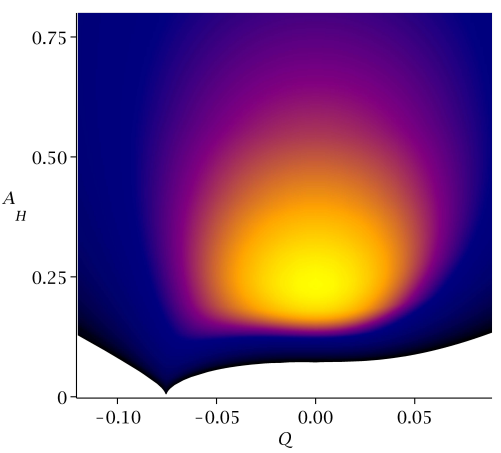

(c) $\lambda=1.5$

Figure 3: Horizon area $A_{H}$ vs. electric charge $Q$ with different temperatures $T_{H}$ for black holes with fixed angular momentum $J=0.00296$ and $L=1$, for $\lambda=0.5$ (a), SUGRA $\lambda=1$ (b), and $\lambda=1.5$ (c). The lower bound of the area is given by the set of $T=0$ extremal solutions. Extremal solutions with negative electric charge possess a critical solution with $A_{H}=0$ at $Q=Q_{0}$, where $Q_{0}=-0.0522,-0.0659,-0.0755$ for $\lambda=0.5$, SUGRA and $\lambda=1.5$, respectively.

solution (essentially, increasing $\lambda$, leads to a larger magnitude of the electric charge of this configuration).

In Figure 4 we show the $\left(M, Q ; T_{H}\right)$ plot for the same BHs with $J=0.0295$. Similarly to the horizon area, the mass is minimized in the extremal case. Here, however, the minimum mass is reached for the extremal MP-AdS BH $(Q=0)$. For negative $Q$, two different branches of extremal BHs can be identified at both sides of the critical solution. However, the mass always increases with the absolute value of the electric charge, contrary to what happens for $A_{H}$.

Non-extremal solutions show a similar behavior for the mass as for the horizon area, and we can clearly identify the local maximum of temperature in the MP-AdS set. The mass of the non-extremal solutions with fixed $J$ can be increased to infinity (which also increases the temperature and the horizon area).

The main difference between the solutions with different $\lambda$ is found close to the critical solution. Here we see a jump on the slope of the $M-Q$ curve; this jump becomes more pronounced when increasing the value of the coupling beyond the SUGRA value. Below $\lambda=1$, the Figure is softened (and the general behavior becomes more symmetric in $Q$ ).

Turning now to the horizon quantities, we present in Figure 5 the plot for $\left(J_{H}, Q ; T_{H}\right)$ for the same set of configurations. This plot is interesting because it gives us an idea about the full domain of existence of the global solutions. For instance, consider Figures 5a and 5c. The lower bound of the horizon angular momentum is given by the set of extremal solutions. The upper bound is given by the line of constant $J_{H}=J=0.00296$. This line is reached asymptotically as the mass of the non-extremal BHs is increased to infinity. To better understand these aspects, we present in Figure $5 \mathrm{~b}$ the $3 \mathrm{D}$ plot $\left(J_{H}, Q ; T_{H}\right)$ for solutions in the SUGRA case. Then one can see more clearly that, close to $J_{H}=J=0.00296$, the temperature drops from the local maximum and then it increases again without limit. The mass and the horizon area also increase and the non-extremal $\mathrm{BHs}$ become more and more massive. Moreover, they have almost all the angular momentum stored behind the horizon (hence reaching the limit $J_{H}=J$ ). Note the surface degenerates into a single line at $Q=0, J=J_{H}$, with $T \in[0, \infty)$, where the full set of MP-AdS BHs is recovered for the three considered cases.

Concerning the extremal solutions, it is interesting to note the difference between the $Q>0$ and $Q<0$ cases. For positive electric charge, adding $Q$ to an extremal MP-AdS solution decreases the horizon angular momentum, and it goes to zero as the electric charge goes to infinity. For negative electric charge, however, the two extremal branches present different properties. In the MP branch, the electric charge can be increased only up to the critical solutions with $Q=Q_{0}$, and $J_{H}$ decreases with $|Q|$. At $Q=Q_{0}$, the horizon angular 


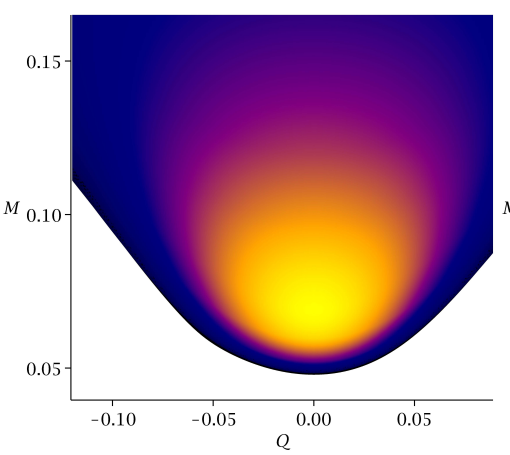

(a) $\lambda=0.5$

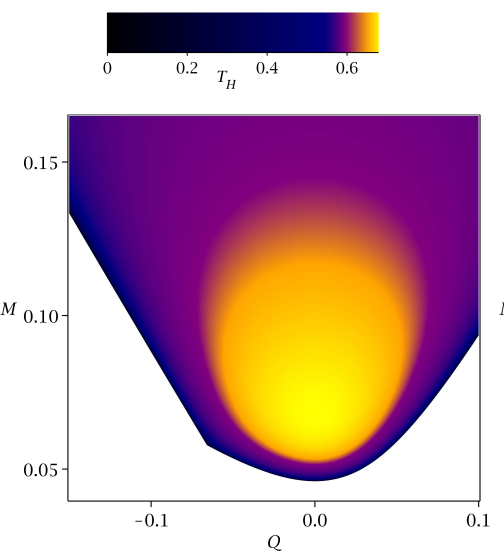

(b) SUGRA

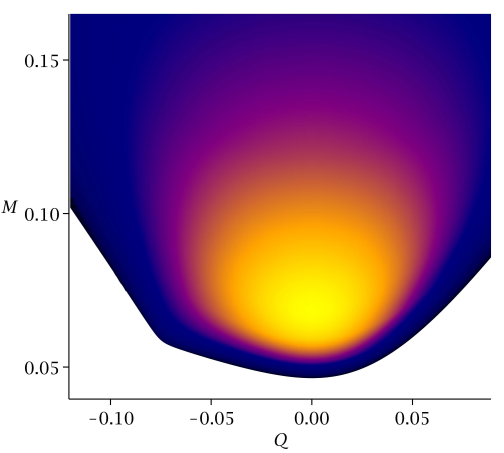

(c) $\lambda=1.5$

Figure 4: Total mass $M$ vs. electric charge $Q$ with different temperatures $T_{H}$ for black holes with fixed angular momentum $J=0.00296$ and $L=1$, for $\lambda=0.5$ (a), SUGRA $\lambda=1$ (b), and $\lambda=1.5$ (c). The lower bound of the mass is given by the set of extremal solutions. The value of the mass of the extremal solutions always increases with the absolute value of the electric charge. The critical solution with zero area at $Q_{0}<0$ can be identified here at the point where the lower bound exhibits a kink.

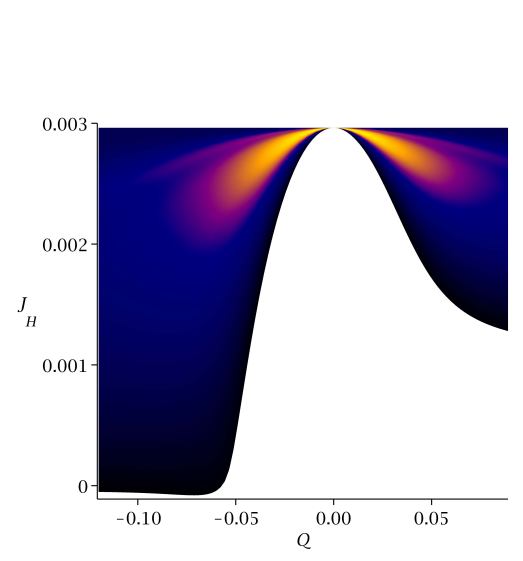

(a) $\lambda=0.5$

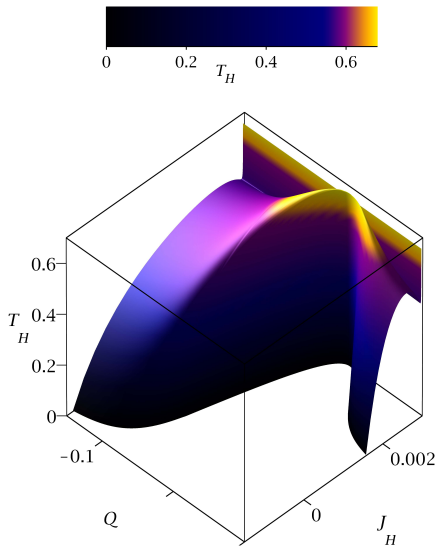

(b) SUGRA

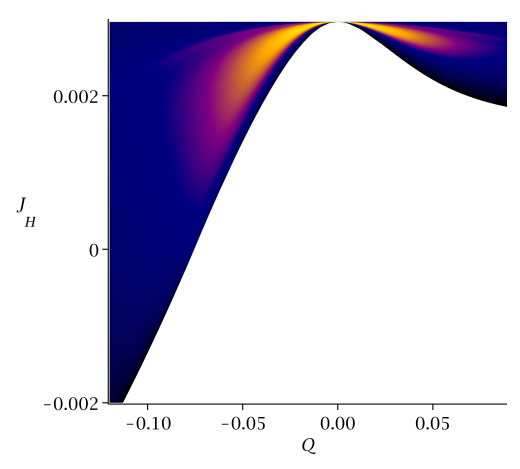

(c) $\lambda=1.5$

Figure 5: Horizon angular momentum $J_{H} v s$. electric charge $Q$ with different temperatures $T_{H}$ for black holes with fixed angular momentum $J=0.00296$ and $L=1$, for $\lambda=0.5$ (a), SUGRA $\lambda=1$ (b), and $\lambda=1.5$ (c). Note that the lower bound of the horizon angular momentum is given by the set of extremal solutions. But now we can see an upper bound, at the line $J_{H}=J=0.00296$. This line is reached asymptotically as the mass and temperature of the non-extremal black holes are increased. The critical solution with zero area at $Q_{0}<0$ can be identified here at the point where the horizon angular momentum is zero. 


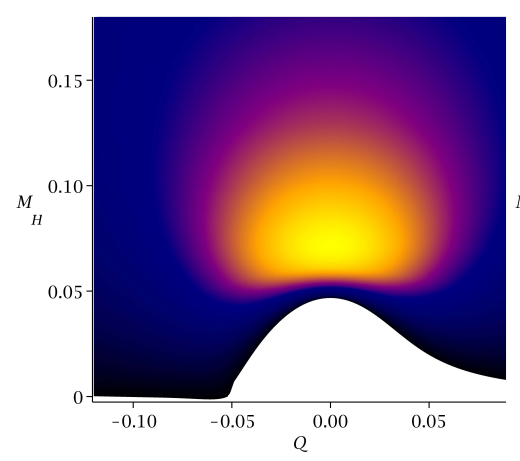

(a) $\lambda=0.5$

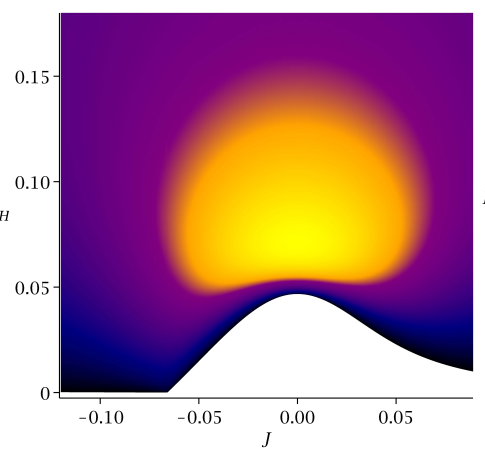

(b) SUGRA

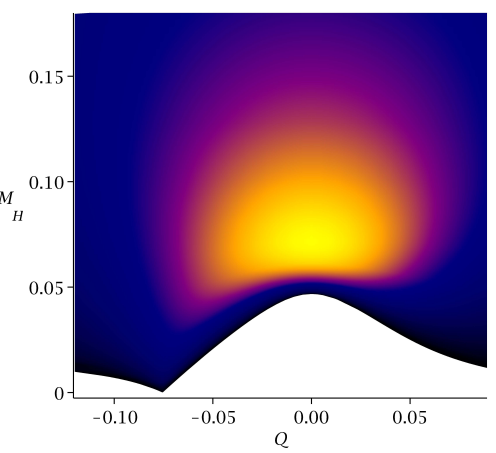

(c) $\lambda=1.5$

Figure 6: Horizon mass $M_{H}$ vs. electric charge $Q$ with different temperatures $T_{H}$ for black holes with fixed angular momentum $J=0.00296$ and $L=1$, for $\lambda=0.5$ (a), SUGRA $\lambda=1$ (b), and $\lambda=1.5$ (c). The main difference between these values of $\lambda$ is in the horizon mass of the extremal and near extremal configurations. In particular the difference is found in the RN branch: note that in (a), when $Q<-0.0522$ the horizon mass can be negative; in (b) when $Q<Q_{0}=-0.0659$ it is always zero; in (c) when $Q<-0.0755$ it is positive and increases with the absolute value of the electric charge.

momentum vanishes. Combining this result with the $Q>0$ case, one can say that the horizon angular momentum of extremal solutions with $Q \in\left(Q_{0}, \infty\right)$ satisfies the relation $0<J_{H} \leq J$, saturating the relation only at $Q=0$.

Another interesting feature one can notice in Figure 5 is the existence of counter-rotating configurations (this holds for all considered values of $\lambda$ ). For example, take the extremal solutions on the RN branch $\left(Q<Q_{0}\right)$ : they have $J>0$, however $J_{H}$ is negative. Moreover, this is not unique to extremal solutions: BHs with $T_{H}>0$ can also become counter-rotating for low enough temperatures and $Q<Q_{0}$.

Changing the coupling $\lambda$ has a particularly relevant effect on these counter-rotating configurations. Note that in the Figure we can see how reducing the coupling below SUGRA reduces the size of the space of solutions with counter-rotation (we will comment on this again in the following sections).

We continue with Figure 6, where we show the $\left(M_{H}, Q ; T_{H}\right)$ plot. One interesting feature of the $\lambda=0.5$ set is the existence of solutions with negative horizon masses. In the extremal case, this happens for solutions on the RN branch and $Q<Q_{0}$. Also, some non-extremal solutions close to this set share the same property. Moreover, one can notice that the horizon mass of the critical solution is always zero, independently of the value of $\lambda$. Surprisingly, in the SUGRA case, the extremal solutions on the RN branch $\left(Q<Q_{0}\right)$ always have $M_{H}=0$, as one can see in Figure $6 \mathrm{~b}$

Hence the main effect of changing the coupling in this case is on the behavior of the horizon mass of the $Q<Q_{0}$ solutions: below SUGRA we can find $M_{H}<0$ configurations, and beyond SUGRA the horizon mass is positive. SUGRA is a very particular case in which $Q<Q_{0}$ extremal black holes have $M_{H}=0$.

In Figure 7 we present the $\left(\Omega_{H}, Q ; T_{H}\right)$ diagram for the same configurations. An interesting behavior occurs here for large enough values of $\lambda$. For example, as seen in Figure $7 \mathrm{~b}$ for the SUGRA case, the extremal BHs on the RN branch $\left(Q<Q_{0}\right)$ always have $\Omega_{H}=0$. However, solutions on the MP branch have positive angular velocity. This indicates the existence of a discontinuity in the angular velocity at the critical solution. This discontinuous behavior is also present for $\lambda=1.5$, see Figure $7 \mathrm{c}$. Along the RN branch $\left(Q<Q_{0}\right)$, the BHs have a counter-rotating horizon: the horizon angular velocity is negative, despite the total angular momentum being positive. However, extremal BHs on the MP branch have positive angular velocity. Hence, one again sees a discontinuous behavior in the angular velocity. Note that non-extremal solutions are not discontinuous: both extremal branches can be joined by almost extremal solutions, for which very small variations of the electric charge cause very large (but continuous) modifications of the angular velocity, and 


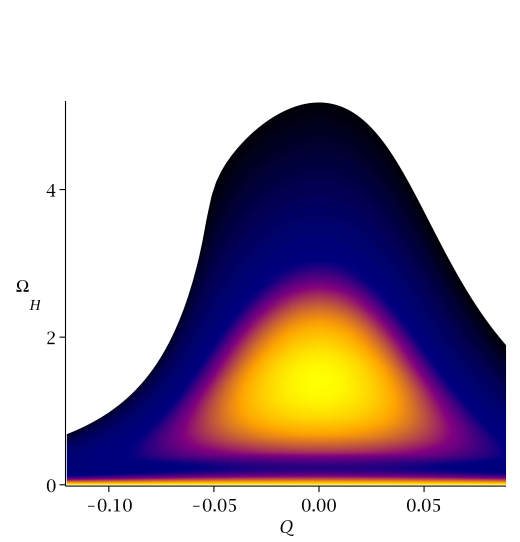

(a) $\lambda=0.5$
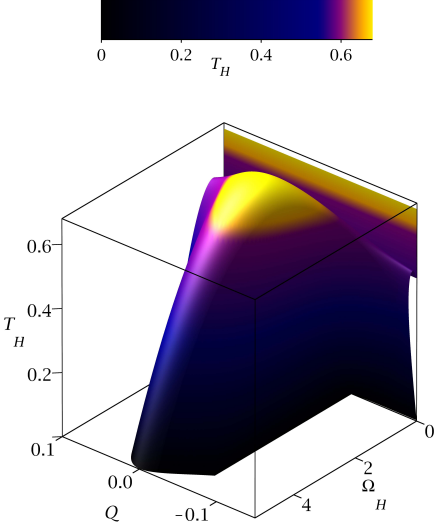

(b) SUGRA

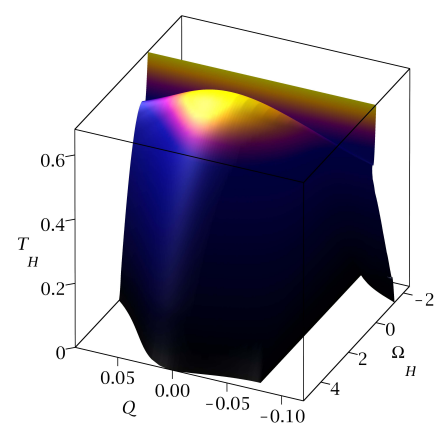

(c) $\lambda=1.5$

Figure 7: Horizon angular velocity $\Omega$ vs. electric charge $Q$ with different temperatures $T_{H}$ for black holes with fixed angular momentum $J=0.00296$ and $L=1$, for $\lambda=0.5$ (a), SUGRA $\lambda=1$ (b), and $\lambda=1.5$ (c). The three cases present very different properties in their extremal limit. Note that $\Omega$ for extremal solutions in $\lambda=0.5$ (a) is continuous. This is no longer the case in SUGRA $\lambda=1$ (b), and $\lambda=1.5$ (c), where the RN branch has $\Omega=0$ and $\Omega<0$ respectively. Since the MP branch has always positive $\Omega$, there is a discontinuity around the critical solution in the angular velocity in the $T=0$ limit for these two cases.

can even change the sense of rotation for big values of $\lambda$.

In these Figures for the angular velocity we can see that once again changing the CS coupling has a very important effect on the properties of the solutions, in particular for $Q<Q_{0}$. SUGRA is a special case where these solutions have null angular velocity, but increasing the value $\lambda$ beyond SUGRA makes this set counter-rotate in the angular velocity.

Finally, in Figure 8 we show the electrostatic potential $\Phi_{H}$ vs. the electric charge $Q$ and the temperature $T_{H}$. In this case, the boundary of the domain of existence is characterized by the set of extremal solutions, and the line $\Phi=0$, which is reached as we move away from extremality by increasing the mass and the temperature.

Similarly to what happens in the angular velocity, changing the coupling $\lambda$ has a specially relevant effect on the $Q<Q_{0}$ solutions. While in the SUGRA case the electrostatic potential is discontinuous and jumps to a larger value when moving from $Q>Q_{0}$ to $Q<Q_{0}$, our calculations show that in non-SUGRA the behavior is softened around $Q=Q_{0}$.

Let us now summarize the main features of these configurations with fixed (positive) total angular momentum.

- For any $\lambda$, there is an asymmetry between solutions with positive and negative electric charges. For positive CS couplings, the $Q<0$ set possesses a critical solution with charge $Q_{0}$, which separates two different extremal branches. This feature is absent for $Q>0$. The critical solution has $A_{H}=M_{H}=$ $J_{H}=0$, and is approached with a discontinuity in both $\Omega_{H}$ and $\Phi_{H}$. An analytical understanding of this behavior is given in Appendix A2, for $\lambda=1$. Also, note that the value of $\left|Q_{0}\right|$ increases as $\lambda$ becomes larger.

- Extremal solutions with $Q<Q_{0}$ present very different horizon properties depending on the value of the CS coupling. For instance, in SUGRA this branch possesses $M_{H}=\Omega_{H}=0$, and separates the $\lambda<1$ case, with $M_{H}<0, \Omega_{H}>0$, from the $\lambda>1$ case, where the sign changes to $M_{H}>0, \Omega_{H}<0$.

- Non-extremal black holes with $Q<Q_{0}$ can possess a counter-rotating horizon $\left(J_{H}<0\right)$, but the size of this set of solutions contracts when decreasing $\lambda$. 


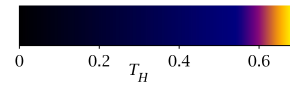

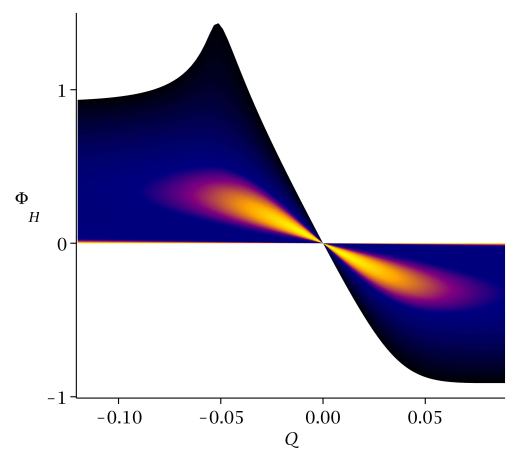

(a) $\lambda=0.5$

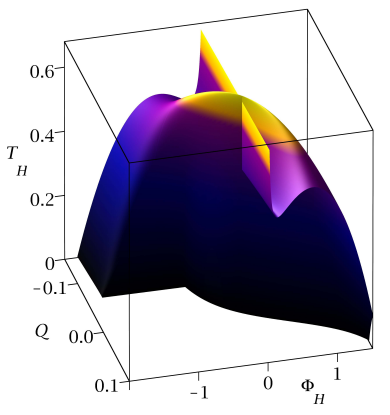

(b) SUGRA

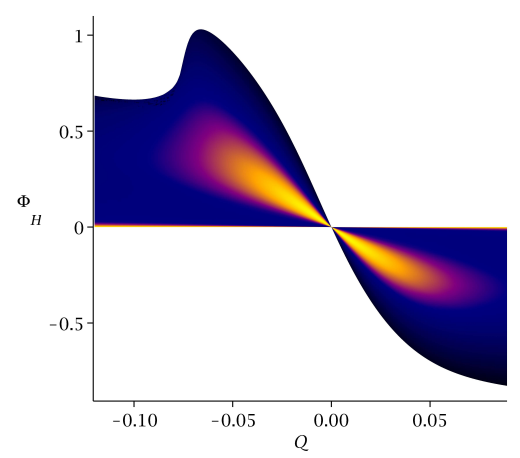

(c) $\lambda=1.5$

Figure 8: Electrostatic potential $\Phi_{H} v s$. electric charge $Q$ with different temperatures $T_{H}$ for black holes with fixed angular momentum $J=0.00296$ and $L=1$, for $\lambda=0.5$ (a), SUGRA $\lambda=1$ (b), and $\lambda=1.5$ (c). The behavior of the electrostatic potential is similar to the angular velocity, with the main differences being in the extremal and near extremal situation.

- Black holes with $\Omega_{H}<0$ can be found for $\lambda>1$. In particular, and since $\Omega_{H}$ is discontinuous in the extremal branch, around the critical solution one can see that small changes in the electric charge causes large variations of the horizon angular velocity, even changing the direction of rotation. On the contrary, for $\lambda<1$ the angular velocity is always in the direction of the angular momentum.

\subsubsection{A fixed electric charge: the generic picture}

A complementary picture is found when fixing the electric charge and varying both $J$ and $T_{H}$. The counterparts of the plots in the previous Subsection are shown in Figures 9 , 14 , for a fixed electric charge $Q=-0.044$ and the same values of $\lambda$.

Again, the global extremal solutions possess two different branches: the MP branch and the RN branch, which agree with the prediction from the near-horizon formalism. The RN branch contains the static configuration with $J=0$, and extends for $J \in\left(-J_{0}, J_{0}\right)$. At $J= \pm J_{0}$ we find the critical solution with $A_{H}=0$. The MP branch connects with the uncharged and rotating black hole, and is found for $|J|>\left|J_{0}\right|$. In what follows, we shall present results for positive values of the angular momentum only, since all properties are symmetric under a change of sign in $J$.

We start with the Figure 9, where we show the $\left(A_{H}, J ; T_{H}\right)$ diagram. The lower boundary of the $A_{H^{-}}$ domain is found for the extremal solutions. Hence, one can say that the $T_{H}=0$ configurations possess the lowest possible horizon area, like in the constant $J$ case. At $J=0$ we find the set of RN-AdS BHs. Note, however, that the extremal RN-AdS solutions do not have the minimum possible entropy. Spinning up an extremal RN-AdS BH while keeping $T_{H}=0$ makes the area to decrease down to zero, a point where the critical solutions are reached. These critical solutions separate the extremal branch originating in the RN solution $\left(0 \leq J<J_{0}\right)$ from the extremal branch connecting with the MP solution $\left(J>J_{0}\right)$. Moreover, although in the RN branch, the horizon area decreases with the angular momentum, in the MP branch $A_{H}$ increases with $J$. An interesting consequence here, is that, for given $Q$, it is possible to obtain EMCS non-extremal spinning and charged BHs with a horizon area lower than the area of the extremal RN-AdS $\mathrm{BH}$.

Concerning the non-extremal solutions, in Figure 9 we can see that the solutions present a local maximum of the temperature. This local maximum is always found for $J=0, T_{H}=0.73$. For higher values of the area, the temperature increases again (not displayed in these Figures). For instance, in the three cases there is no upper bound for the entropy. Also, one can see in Figure 9 that it is possible to define closed sets of 


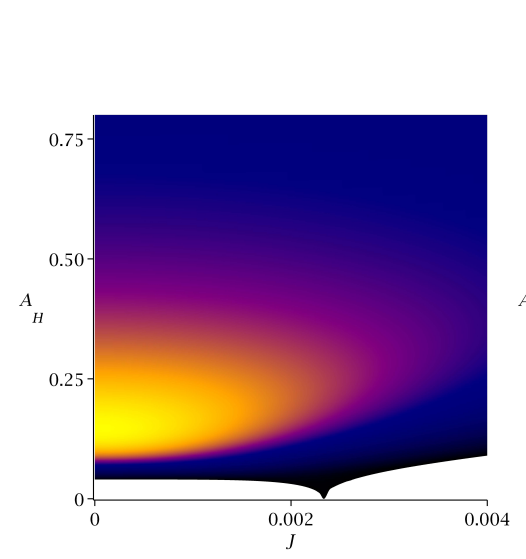

(a) $\lambda=0.5$

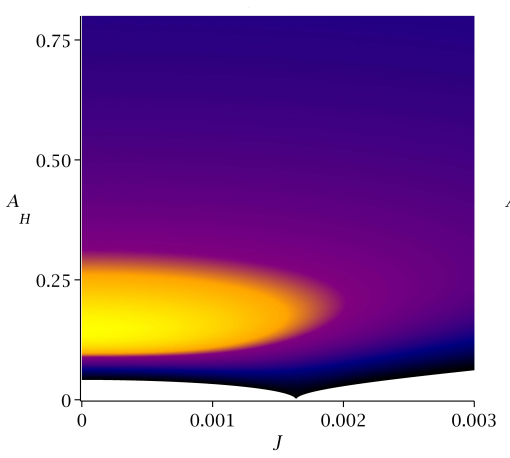

(b) SUGRA

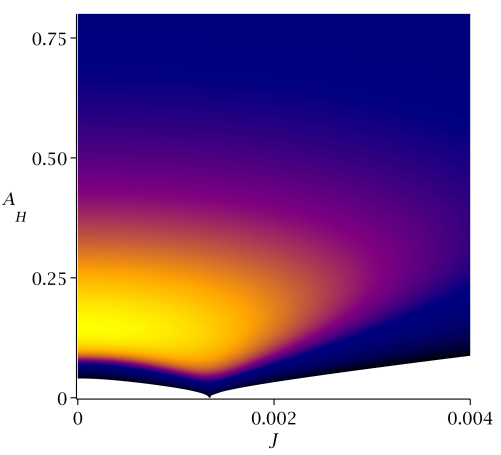

(c) $\lambda=1.5$

Figure 9: Horizon area $A_{H}$ vs. angular momentum $J$ with different temperatures $T_{H}$ for black holes with fixed electric charge $Q=-0.044$ and $L=1$, for $\lambda=0.5$ (a), SUGRA $\lambda=1$ (b), and $\lambda=1.5$ (c). The lower bound is given by the set of extremal solutions. The critical solution with $A_{H}=0$ is found at $J=J_{0}$, where $J_{0}=0.00233,0.00164,0.00133$ for $\lambda=0.5$, SUGRA and $\lambda=1.5$, respectively.

isothermal rotating and charged BHs.

The main effect of changing the CS coupling is on the value of $J_{0}$ : increasing $\lambda$ reduces $J_{0}$ and shrinks the extremal RN branch.

In Figure 10 we show the $\left(M, J ; T_{H}\right)$ plot. The local maximum temperature is obtained for a non-extremal RN-AdS configuration $(J=0)$. The existence of this local maximum of temperature implies the existence of a set of thermally unstable configurations with negative thermal capacity. Around this set, isothermal sets are closed. This means that the mass-angular momentum relation is bounded. This is not the case for isothermal sets far away from the local maximum.

The extremal solutions present very different behavior depending on the value of $\lambda$ : For $\lambda=0.5$ (Figure 10a), we can see that extremal BHs on the RN branch $\left(J<J_{0}\right)$ have total mass increasing with the angular momentum. This means that the minimum mass is obtained for the extremal RN-AdS BH. Extremal BHs along the MP branch $\left(J>J_{0}\right)$ have total mass increasing with the angular momentum. For $\lambda=1$ (Figure 10b), this is different: all extremal BHs on the RN branch have the same (minimal) mass. The mass only starts increasing when $J>J_{0}\left(J_{0}=0.00164\right)$. Again, extremal BHs along the MP branch have the total mass increasing with the angular momentum. For $\lambda=1.5$ (Figure 10c this changes again, and extremal BHs on the RN branch $\left(J<J_{0}\right)$ have the total mass decreasing with the angular momentum. But extremal $\mathrm{BHs}$ along the MP branch have the total mass increasing with the angular momentum. This means the minimum mass is reached at the critical solution with $J_{0}=0.00133$.

This situation contrasts with the one presented in Figure 3 for the fixed $J$ case, where the minimum mass was always found at the extremal uncharged solution. Here it clearly depends on the value of the CS coupling being below or beyond the SUGRA case.

In Figure 11 we show the diagram for $\left(J_{H}, J ; T_{H}\right)$. One can see that the upper bound there is given by the line $J_{H}=J$. These solutions are reached as the mass of the BHs increases. This means that all the angular momentum of these solutions is stored behind the horizon, with a vanishing contribution from the gauge field. The lower bound contains the extremal solutions. In these three cases, the critical solution with fixed $Q$ has $J_{H}=0$. Extremal $\mathrm{BHs}$ on the RN branch always present negative horizon angular momentum. However, extremal $\mathrm{BHs}$ on the MP branch have positive horizon angular momentum, and it is approximately given by $J_{H} \approx J-J_{0}$.

The RN branch having negative horizon angular momentum means the solutions are counter-rotating, a feature which, in fact, is also shared by whole sets of non-extremal BHs. Even more, one can find nonextremal solutions with zero horizon angular momentum, but non-zero total angular momentum. For such 


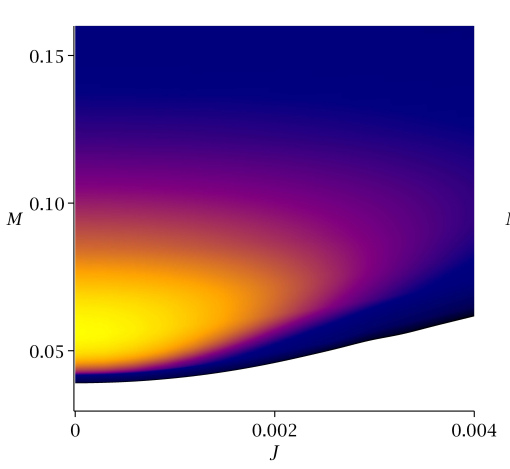

(a) $\lambda=0.5$

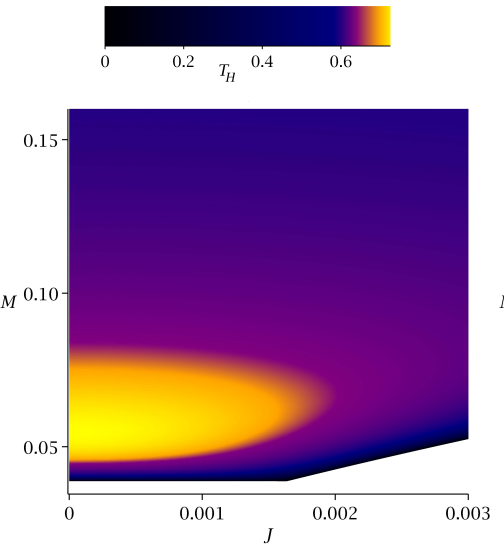

(b) SUGRA

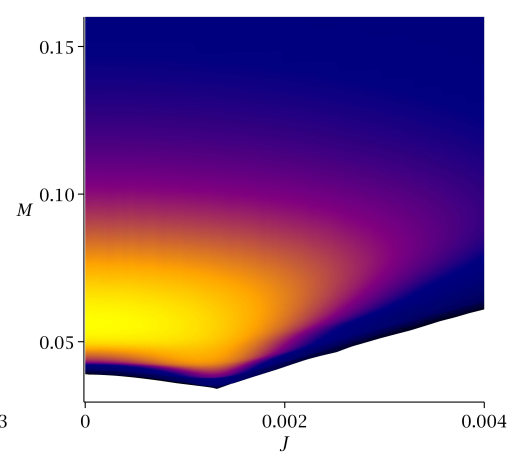

(c) $\lambda=1.5$

Figure 10: Total mass $M v s$. angular momentum $J$ with different temperatures $T_{H}$ for black holes with fixed electric charge $Q=-0.044$ and $L=1$, for $\lambda=0.5$ (a), SUGRA $\lambda=1$ (b), and $\lambda=1.5$ (c). Note that the properties of the extremal set (lower boundary) depend on the CS coupling. When $\lambda \geq 1$, the extremal RN solution is no longer the black hole with minimum mass.

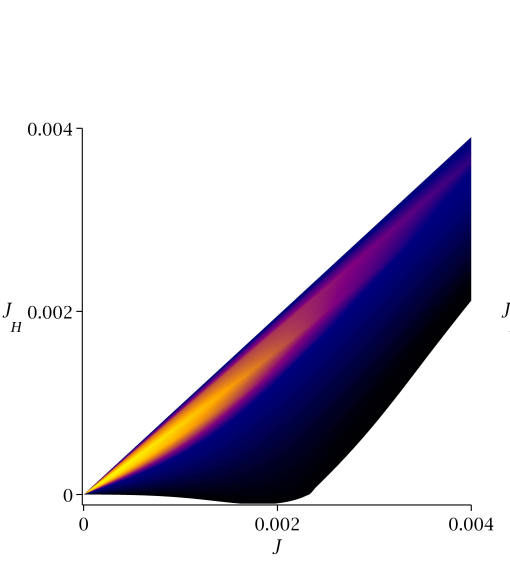

(a) $\lambda=0.5$

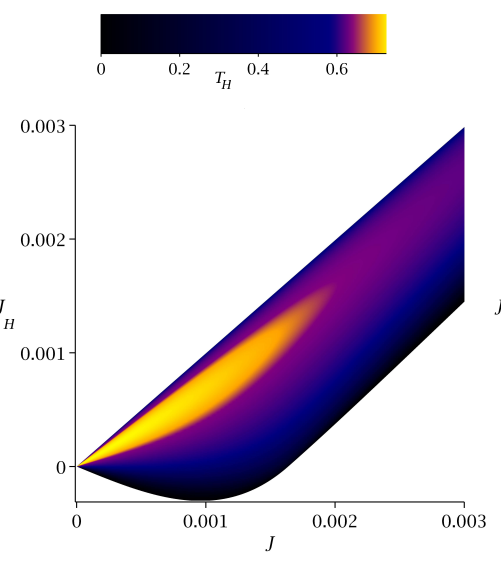

(b) SUGRA

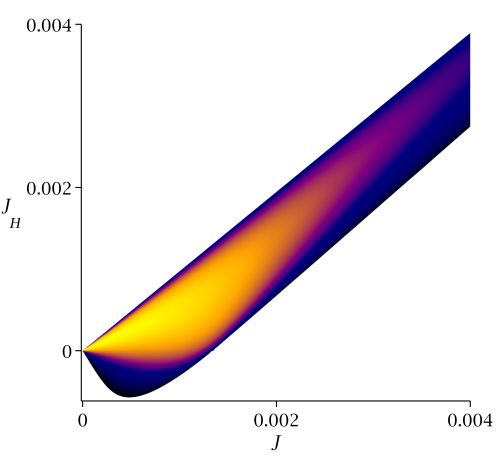

(c) $\lambda=1.5$

Figure 11: Horizon angular momentum $J_{H}$ vs. angular momentum $J$ with different temperatures $T_{H}$ for black holes with fixed electric charge $Q=-0.044$ and $L=1$, for $\lambda=0.5$ (a), SUGRA $\lambda=1$ (b), and $\lambda=1.5$ (c). Note that we can find extremal and non-extremal configurations with $J_{H}<0$. Extremal black holes on the MP branch approximately satisfy $\left(J_{H} \approx J-J_{0}\right)$. 


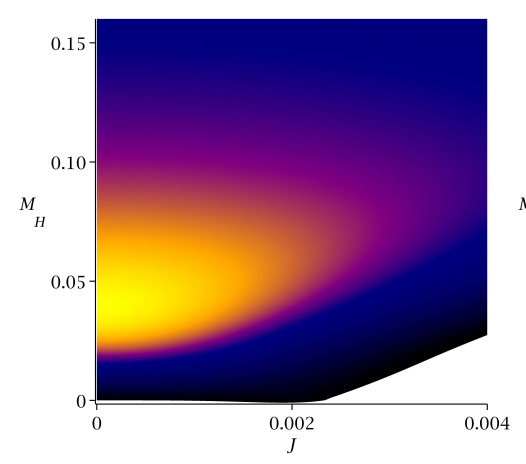

(a) $\lambda=0.5$

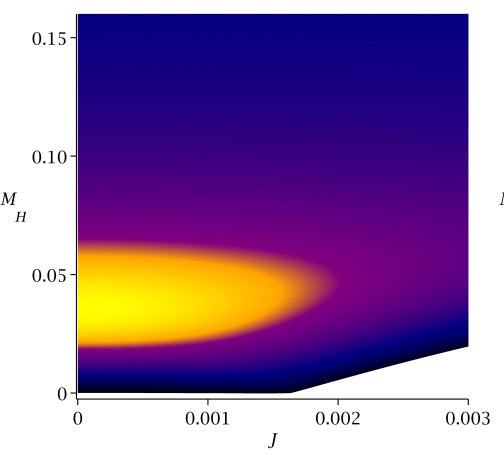

(b) SUGRA

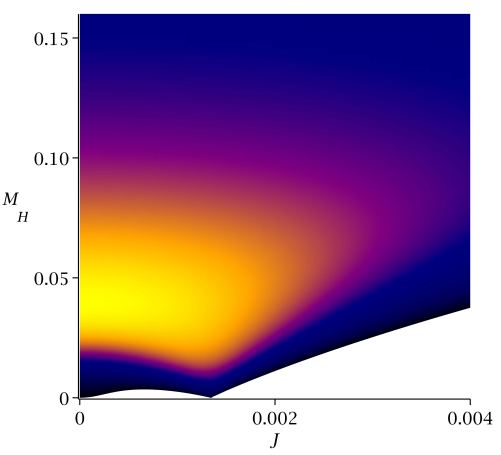

(c) $\lambda=1.5$

Figure 12: Horizon mass $M_{H}$ vs. angular momentum $J$ with different temperatures $T_{H}$ for black holes with fixed electric charge $Q=-0.044$ and $L=1$, for $\lambda=0.5$ (a), SUGRA $\lambda=1$ (b), and $\lambda=1.5$ (c). Note SUGRA is a particular case in which the RN-branch has always $M_{H}=0$. This feature is lost when changing the value of the coupling.

solutions, the angular momentum is stored in the gauge field.

The variation of the coupling $\lambda$ has an important effect on these counter-rotating configurations: reducing the coupling below SUGRA again reduces the size of the space of solutions with counter-rotation.

In Figure 12 we present the $\left(M_{H}, J ; T_{H}\right)$ plot. The minimal value of the horizon mass if reached for extremal solutions. The static extremal MP-AdS BH at $J=0$ has zero horizon mass. In the three cases, the horizon mass of the critical solution is also zero. But the properties close to extremality of the non-static solutions depend considerably on the value of $\lambda$. As seen in Figure 12a, the solutions with $J<J_{0}$ (extremal solutions on the RN branch or near extremal solutions close to it) can have negative horizon mass. However, one can see in Figure 12b that this is no longer the case for SUGRA BHs, and the extremal RN branch has always zero horizon mass. In Figure 12c, we can see that the RN branch has positive horizon mass when $\lambda=1.5$. In fact it is interesting to note that the horizon mass of the extremal RN branch increases up to a maximum, and then it decreases again to zero.

In Figure 13 we show the $\left(\Omega_{H}, J ; T_{H}\right)$ diagram. The differences there occur especially close to extremality. As seen in Figure 13a, the angular velocity of the extremal RN branch is positive, and matches with the angular velocity of the extremal MP branch. Consider now the CPL solution in Figure 13b. Note that there is a discontinuity in the angular velocity at zero temperature: the extremal BHs connecting with the RN solution have zero angular velocity. At the critical solution, the angular velocity jumps up to a positive value, where the MP branch starts. Then the angular velocity decreases with the angular momentum. The $\lambda=1.5$ case (Figure 13c), also presents a discontinuity in the angular velocity. New features occur here as well. For example, note that the horizon angular velocity of the extremal RN branch is negative. This means one finds counter-rotating solutions. Such BHs can be non-extremal too. Also, note that the angular velocity of non-extremal solutions close to the critical solution presents very steep changes with respect to small changes in the angular momentum. Hence, if one perturbs the angular velocity of one of these solutions slightly, the angular velocity and even the direction of the rotation can change drastically. Nevertheless, other quantities (e.g. mass and horizon area) do not change much.

In Figure 14 we show the $\left(\Phi, J ; T_{H}\right)$ diagram. Again, the properties of the extremal solutions depend on the particular value of the CS coupling $\lambda$, although the features of BHs far from extremality are rather similar. Note that in the CPL solution, Figure 14b, on the branch connecting with the MP BH, the electric potential depends on the angular momentum, while on the branch connecting with the RN solution, the electric potential is constant. In the other two cases, Figure 14a and Figure 14c the particular dependence of $J$ on the electrostatic potential on the RN branch depends on the coupling $\lambda$. 


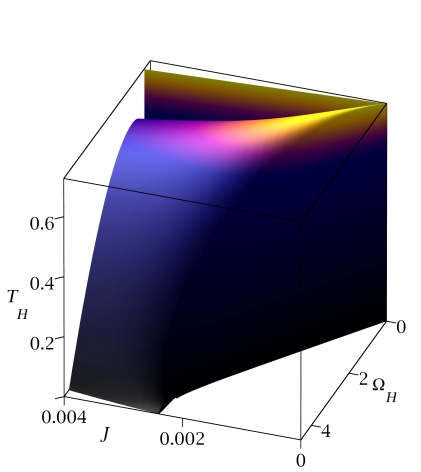

(a) $\lambda=0.5$

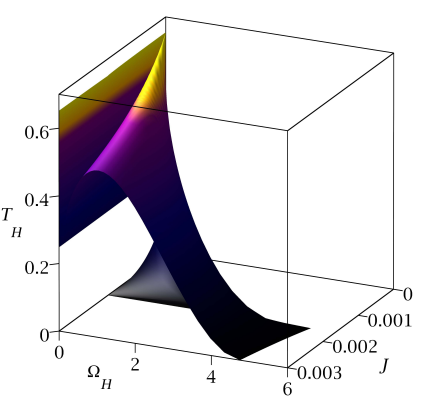

(b) SUGRA

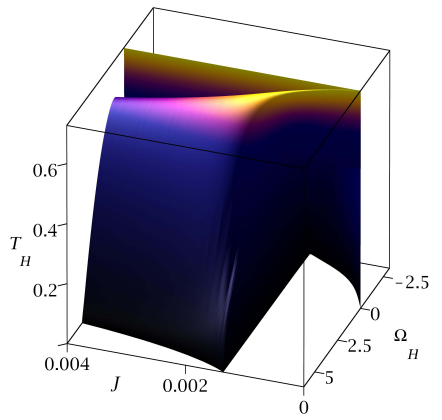

(c) $\lambda=1.5$

Figure 13: Horizon angular velocity $\Omega_{H} v s$. angular momentum $J$ with different temperatures $T_{H}$ for black holes with fixed electric charge $Q=-0.044$ and $L=1$, for $\lambda=0.5$ (a), SUGRA $\lambda=1$ (b), and $\lambda=1.5$ (c). The extremal limit has very different properties in each case. Note that $\Omega_{H}$ for extremal solutions in $\lambda=0.5$ (a) is continuous, but discontinuous in the other two cases. For SUGRA $\lambda=1$ (b), and $\lambda=1.5$ (c), the RN branch has $\Omega_{H}=0$ and $\Omega_{H}<0$, respectively, and the MP branch has always positive $\Omega_{H}$. This is equivalent to Figure 7

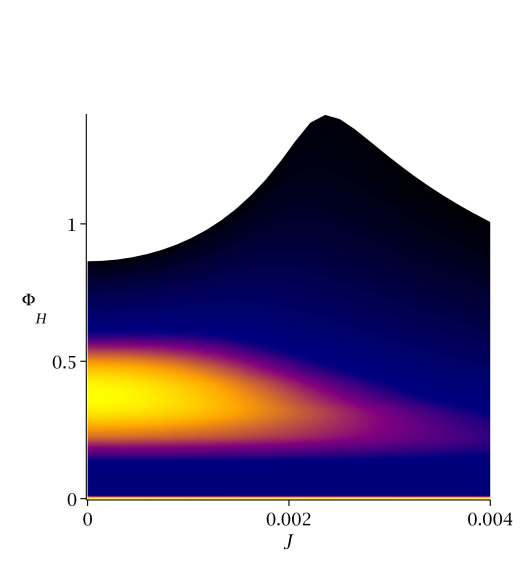

(a) $\lambda=0.5$

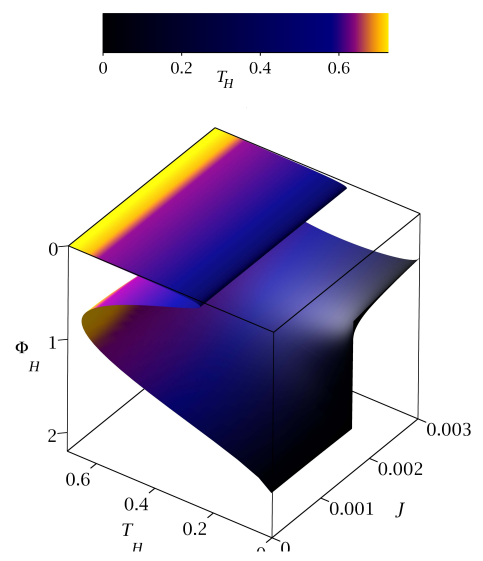

(b) SUGRA

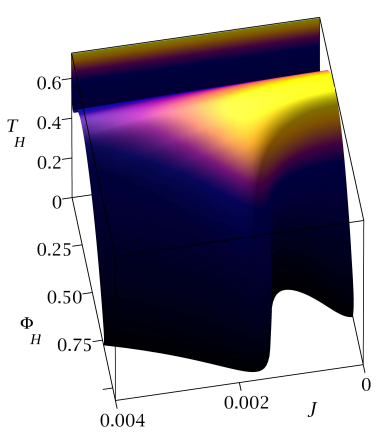

(c) $\lambda=1.5$

Figure 14: Electrostatic potential $\Phi_{H}$ vs. angular momentum $J$ with different temperatures $T_{H}$ for black holes with fixed electric charge $Q=-0.044$ and $L=1$, for $\lambda=0.5$ (a), SUGRA $\lambda=1$ (b), and $\lambda=1.0$ (c). 


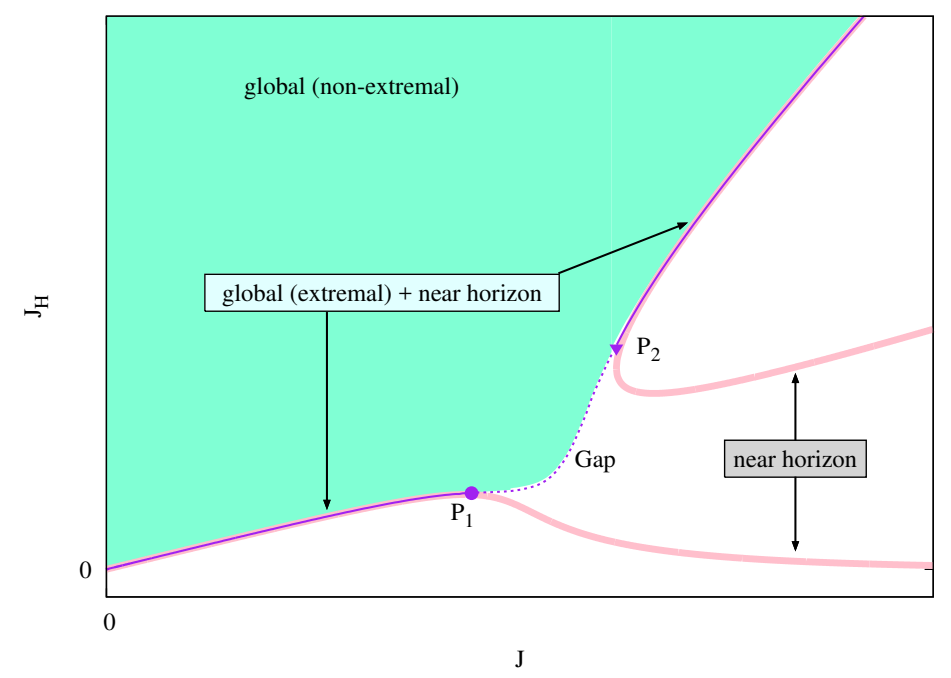

Figure 15: The generic relation between near-horizon and global solutions found for small enough values of $\lambda$ is shown in a $\left(J_{H}, J\right)$-diagram.

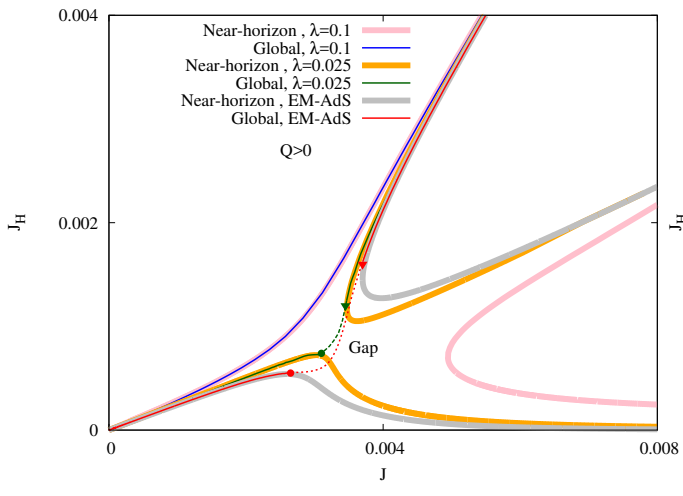

(a) $Q>0$

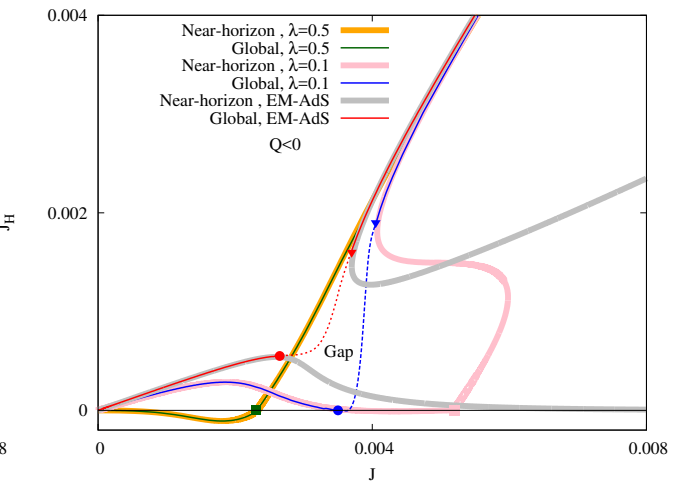

(b) $Q<0$

Figure 16: Horizon angular momentum $J_{H}$ vs. angular momentum $J$ for extremal black holes with fixed electric charge $Q=|0.044|$ and $L=1$, for small values of $\lambda$. Thick curves in grey, orange and pink represent near-horizon solutions. Thin curves in red, blue and green represent global extremal black holes. The corresponding dashed curves represent near-extremal solutions. The dots and triangles represent the limiting solutions separating the extremal branches, and the squares the critical solutions. One can see that not all near-horizon solutions correspond to a global configuration. 
Finally, let us summarize the main features of the configurations with fixed negative electric charge.

- For every $\lambda$, one can find a critical solution with angular momentum $J_{0}$, which separates two different extremal branches: the RN branch with $J<J_{0}$ and the MP branch with $J>J_{0}$. The critical solution has $A_{H}=M_{H}=J_{H}=0$, and a discontinuity in $\Omega_{H}$ and in $\Phi_{H}$. The value of $J_{0}$ decreases with increasing $\lambda$.

- The RN branch properties depend on the value of the CS coupling. For instance, in the SUGRA case the RN branch possesses a constant mass, but for $\lambda<1$ the mass increases monotonically with $J$, while for $\lambda>1$ it decreases. For SUGRA, the RN branch satisfies $M_{H}=\Omega_{H}=0$, while for $\lambda<1$ one finds $M_{H}<0, \Omega_{H}>0$, and for $\lambda>1$ the behavior changes to $M_{H}>0, \Omega_{H}<0$.

- Non-extremal black holes with $J<J_{0}$ can present counter-rotation $\left(J_{H}<0\right)$. The size of this set reduces when we decrease $\lambda$.

- Similar to BHs with fixed $J, \Omega_{H}<0$ can be found only for $\lambda>1$, and the angular velocity of nonextremal BHs can change abruptly around the critical solution under small changes of the angular momentum, affecting even the direction of rotation.

\subsection{Solutions with a small Chern-Simons coupling}

We have studied also families of solutions with values of CS coupling constant $\lambda<0.5$, which was the minimal considered value in the previous Subsection. We recall that the attractor solutions in Section 3 predict in this case the existence of new features of extremal BHs, with a bifurcating branch structure. Indeed, our numerical results for global solutions show that this is the case and the picture discussed in the Section 5.1 fails to capture some properties of the BHs with small enough $\lambda$. This holds in particular for extremal $\mathrm{BH}$ solutions, whose study will allow us to better understand how generic the predictions of the near-horizon formalism in Section 3 are.

The generic picture found in this case is shown in Figure 15, for a $\left(J_{H}, J\right)$-diagram of solutions with a fixed $Q$. Both near-horizon and global solutions are shown there. One can see that, starting at $J=J_{H}=0$ extremal RN-AdS solutions, one finds a branch of BHs that ends at a limiting solution with some nonzero values of $J, J_{H}$ (the point $P_{1}$ ). A second branch of global extremal $\mathrm{BHs}$ is found coming from large values of $J, J_{H}$, and ending at the point $P_{2} \neq P_{1}$. Note that these two branches are in agreement with parts of the branches predicted by the near-horizon formalism. The points $P_{1}$ and $P_{2}$ are connected by a particular set of extremal solutions (blue dashed line), which is called in what follows the gap set. These solutions emerge as the limit of near-extremal global configurations and appear to possess some pathological properties. For example, the Kretschmann scalar takes very large values at the horizon, which makes the direct construction of the extremal solutions connecting $P_{1}$ and $P_{2}$ difficult. The global non-extremal solutions exist in a domain bounded below by the global extremal BHs connected by the gap set. Another important feature one can see in Figure 15 is that a part of the near-horizon solutions do not have global counterparts.

Numerical results supporting the above picture are shown in Figure 16, where we consider extremal solutions with a fixed electric charge $Q=|0.044|$ and several values of $\lambda$ (qualitatively similar pictures have been found for other values of $Q$ ). The case $Q>0$ is shown in Figure 16a. The grey, orange and pink curves there correspond to near-horizon solutions with $\lambda=0, \lambda=0.025$ and $\lambda=0.1$ respectively. Note that these solutions have been presented already in Section 3, where we have noticed the existence of two different branches of near-horizon solutions for small enough values of $\lambda$, (in particular for $\lambda=0$ and $\lambda=0.025$ ), which bifurcate at $\lambda=0.0305$. In the same Figure $16 \mathrm{a}$ we add the corresponding sets of global extremal solutions (for example, the red thin line represents global extremal BHs in pure EM-AdS theory). A similar structure is found for other small enough values of the CS coupling constant, in particular for $\lambda=0.025$, see Figure 14. One can see that when $\lambda>0.0305$ the space of configurations no longer presents a gap set. For instance, consider in Figure 16a the blue line. This is the set of global extremal solutions with $\lambda=0.1$, and it matches perfectly (in one to one correspondence) with the near-horizon solutions (pink line).

Let us consider now the negative charge case, $Q<0$. Some results in this case are shown in Figure $16 \mathrm{~b}$ One can see that the qualitative picture discussed above for $Q>0$ still holds. For example, consider the pure 


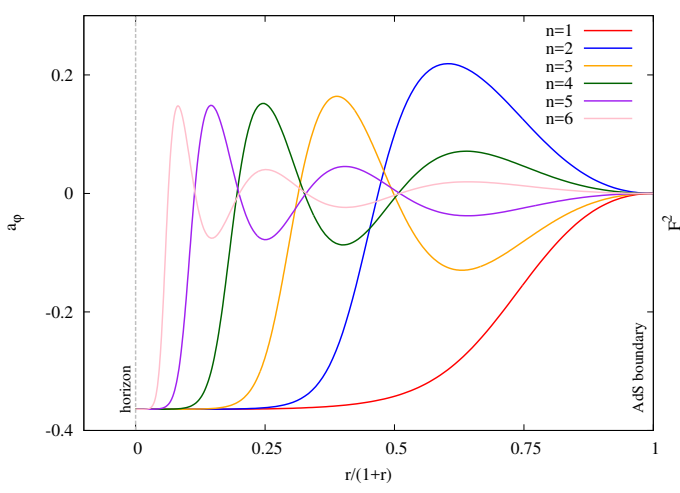

(a)

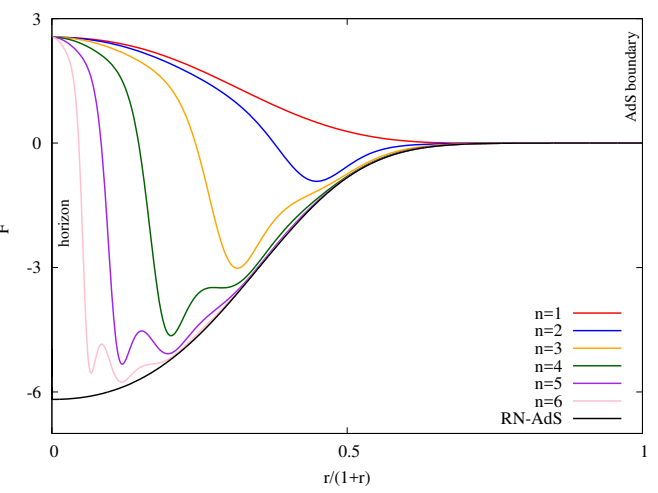

(b)

Figure 17: (a) The profile of the magnetic gauge potential $a_{\varphi}(r)$ is shown vs. the compactified radial coordinate for a sequence of extremal black holes with a different nodal structure. (b) The invariant $F^{2}=$ $F_{\mu \nu} F^{\mu \nu}$ is shown vs. the compactified radial coordinate for the same set of solutions; the corresponding profile for the extremal RNAdS solution is also included for reference. These solutions have $J=0, \lambda=5$, $L=10$ and $Q=-2.72$.

$\lambda=0.1$ case (blue curve). Then one finds again the existence of a gap set (dashed blue line) connecting two disconnected branches of BHs that end at two different limiting solutions (blue dot and blue triangle). As a consequence, not all near-horizon solutions for this value of $\lambda$ (pink line) correspond to global solutions. The critical solution with $J_{H}=0$ cannot be reached for this value of $\lambda$. The situation changes when $\lambda>0.25$, in which case the gap disappears (see the curve for $\lambda=0.5$ ). The two branches of extremal BHs (green line) are now joined at the critical solution with $J_{H}=0$ (green rectangle). Interestingly, in this case with $\lambda=0.5$, all near-horizon solutions (orange line) correspond to a global solution. However, in the other cases, this is not true. Hence we conclude that also for $Q<0$ there are cases for which near-horizon solutions do not correspond to global solutions.

To summarize, the solutions with a small enough value of $\lambda$ show a number of features which are not captured by the knowledge of the CLP BHs (e.g. the existence of a gap set).

\subsection{Large Chern-Simons coupling: discrete sets of radially excited extremal black holes.}

New features occur as well for large enough value of $\lambda$. For example, we have considered solutions with $\lambda=5$ and found an overall picture which is qualitatively similar to the one obtained in the asymptotically flat case 11. The most interesting new feature here is the appearance of a set of non-static extremal BHs with vanishing total angular momentum, $J=0$. This special set contains a large number of distinct solutions, possibly an infinite on $5^{5}$. Its members can be labeled by an integer $n \geq 1$, which can be identified with the the number of nodes found in the profiles of the metric function $\omega(r)$, and of the magnetic gauge potential $a_{\varphi}(r)$. The solutions reported in the previous Section are the fundamental ones, with $n=1$ (since they still possess a zero of $\omega(r), a_{\varphi}(r)$, reached as $\left.r \rightarrow \infty\right)$. The mass of the solutions increases with $n$, the numerics indicating that the extremal RN-AdS BH mass would be approached as $n \rightarrow \infty$. Other quantities, like the horizon angular momentum and horizon angular velocity decrease with the $n$ number.

The profiles of the magnetic gauge potential $a_{\varphi}$ are shown in Figure $17 \mathrm{a}$ for some typical set of $J=0$ extremal BHs with $n=1, \ldots, 6$. The number of nodes leaves an imprint also in the invariant quantities. For example, in Figure $17 \mathrm{~b}$ the square of the gauge field tensor, $F^{2}=F_{\mu} F^{\mu \nu}$, is shown for the same configurations. The 'oscillations' there are found also for the components of the energy-momentum tensor.

\footnotetext{
${ }^{5}$ So far we have constructed solutions with the highest node number $n=40$. However, it is natural to conjecture the existence of solutions with arbitrarily high values of $n$.
} 
As such, the excited solutions $(n>1)$ possess a "layer structure", with $n$ distinct radii where the energy density concentrates. The more nodes, the more layers the solution develops in the bulk.

However, although a number $n>1$ modifies the properties of the BHs in the bulk, the nodal structure is not seen in the near-horizon behavior. That is, in that limit, they are still described by the same squashed $A d S_{2} \times S^{3}$ solutions discussed in Section 3. Then, we conclude that similar to the asymptotically flat case [11, a given near-horizon configuration can correspond to more than one global solution (likely an infinite set).

The non-extremal BHs possess also excitations, in which case, however, we noticed the existence of a maximal value of $n$. Moreover, as expected, excited solutions exist also for $T_{H}=0$ and a nonzero $J$.

A detailed analysis of the excited configurations, with a full study of the branch structure and thermodynamic properties, will be presented elsewhere [43.

Finally, we mention that, unfortunately, no such excited solutions could be found in the special $\lambda=1$ case. In principle, in the absence of a uniqueness proof of the CLP solution, their existence cannot be excluded. However, for all input parameters we have considered so far, the nodal structure disappears for values of $\lambda$ smaller than the SUGRA value.

\section{Conclusions}

This paper has presented a discussion of the basic properties of charged rotating BHs in a $D=5$ EMCSAdS theory with an arbitrary value of the CS coupling constant $\lambda$. The considered solutions have two equal-magnitude angular momenta, possess no pathologies on and outside of an event horizon of spherical topology, and approach at infinity a globally AdS background. So far, the only known solutions of the EMCS-AdS model compatible with these assumptions are the BHs found in [7] by Cvetič, Lü and Pope (CLP) for $\lambda=1$, i.e. a minimal gauged supergravity model. The main questions we have tried to answer were: "How general are the properties of the CLP solution?" and "Are there new features for other values of $\lambda$ ?".

The main conclusion of our study is that the intuition based on the CLP BHs cannot be safely extrapolated to solutions of a generic EMCS model. New unexpected features occur for sufficiently small values of $\lambda$ (in particular for an EM model) and for also for large $\lambda$.

The comparison with the SUGRA exact solutions is most easily done in the extremal case. This limit reduces the parameter space of solutions and also allows for a partial analytical understanding based on results within the attractor mechanism.

For sufficiently small values of $\lambda$ the most interesting new feature (which is absent in the SUGRA case), is the existence of two disconnected branches of extremal BHs. The bridge between these BHs is provided by a set of extremal solutions which appear to possess pathological properties. Also, this gap set cannot be described within the attractor mechanism.

New properties occur as well for large $\lambda$. The most striking one is the existence of excited solutions, which are labeled by the number of nodes $n$ of the metric function $\omega(r)$ (or of the magnetic gauge potential $a_{\varphi}(r)$ ). This nodal structure does not exist in the SUGRA case, where we could not (numerically) find other solutions which in principle could exist apart from the $n=1$ in [7. Also, it cannot be captured by the near-horizon configurations. In fact, the relation between the solutions found within the attractor formalism and the global ones is quite intricate. For example, a given near-horizon solution can correspond to one global solution, more than one global solution (possibly even an infinite set), or, more striking, no global solution at all.

Other, less spectacular differences, which occur when varying $\lambda$, are discussed in the Section 5.2.

However, there are also a number of features which seem to be generic for any $\lambda$. For example, the presence of a CS term always implies the occurrence of a critical set of extremal solutions with a vanishing event horizon area, $A_{H}=0$. Also, the overall thermodynamical behavior of the solutions is well captured by the exact CLP solution, the BHs possessing a positive heat capacity for large enough values of $J, Q$. Moreover, the solutions with large temperatures are less sensitive to changes in $\lambda$. 
The solutions obtained in this paper may provide a fertile ground for the further study of charged rotating configurations in EMCS theory with a negative cosmological constant. For example, their generalization to include more scalars is straightforward. Also, in principle, by using the same techniques, there should be no difficulty to construct similar AdS solutions in $D=2 N+1$ dimensions, with $N>2$ equal-magnitude angular momenta. Also, it would be interesting to find applications of the solutions in this work in an AdS/CFT context. However, the fact that all $\lambda \neq 1$ solutions do not solve a supergravity model makes it more difficult to obtain a CFT description.

\section{Acknowledgements}

J.L.B.S. would like to thank Robert Mann and Carlos Herdeiro for helpful comments and discussions. We gratefully acknowledge support by the DFG Research Training Group 1620 "Models of Gravity". E. R. acknowledges funding from the FCT-IF programme. This work was also partially supported by the H2020MSCA-RISE-2015 Grant No. StronGrHEP-690904, and by the CIDMA project UID/MAT/04106/2013. J.L.B.S. and J.K. gratefully acknowledge support by the grant FP7, Marie Curie Actions, People, International Research Staff Exchange Scheme (IRSES-606096). F. N.-L. acknowledges funding from Complutense University under Project No. PR26/16-20312.

\section{Note added:}

During the preparation of this paper for publication, we received communication from M. Mir and R. B. Mann concerning their research in [44, which overlaps with some of the results presented here. However, the approach in [44] is complementary to that in our work, Mir and Mann presenting closed form solutions obtained via a perturbative approach around the MPAdS BHs.

\section{A The $\lambda=1$ Cvetič-Lü-Pope black holes}

\section{A.1 Solution}

The most general charged rotating BH solution with two equal angular momenta of the EMCS-AdS equations, which is known in closed form, has been reported by Cvetič, Lü and Pope in Ref. 7] (see also [45, 46, 47] for further investigations of it).

The expression of this solution within the Ansatz (4) and (5) reads

$$
\begin{aligned}
& \frac{1}{F_{1}(r)}=1-\frac{2 m\left(1-\frac{a^{2}}{L^{2}}\right)-2 q}{r^{2}}+\frac{2 a^{2} m+\left(1-\frac{a^{2}}{L^{2}}\right) q^{2}}{r^{4}}+\frac{r^{2}}{L^{2}}, \quad F_{2}(r)=r^{2}, \\
& F_{3}(r)=r^{2}\left(1+\frac{2 a^{2} m}{r^{4}}-\frac{a^{2} q^{2}}{r^{6}}\right), \quad F_{0}(r)=\frac{1-\frac{2 m\left(1-\frac{a^{2}}{L^{2}}\right)-2 q}{r^{2}}+\frac{2 a^{2} m+\left(1-\frac{a^{2}}{L^{2}}\right) q^{2}}{r^{4}}+\frac{r^{2}}{L^{2}}}{1+\frac{2 a^{2} m}{r^{4}}-\frac{a^{2} q^{2}}{r^{6}}}, \\
& W(r)=\frac{a\left(2 m-q-\frac{q^{2}}{r^{2}}\right)}{r^{4}\left(1+\frac{2 a^{2} m}{r^{4}}-\frac{a^{2} q^{2}}{r^{6}}\right)}, \quad a_{\varphi}(r)=-\frac{\sqrt{3} a q}{2 r^{2}}, \quad a_{0}(r)=\frac{\sqrt{3} q}{2 r^{2}}
\end{aligned}
$$

where $a, q$ and $m$ are three constants. To make contact with the approach in this work, we express $m$ as a function of the event horizon radius $r_{H}$ (with $1 / F_{1}\left(r_{H}\right)=F_{0}\left(r_{H}\right)=0$ ):

$$
m=\frac{\frac{r_{H}^{4}}{2 L^{2}}+\left(\frac{q+r_{H}^{2}}{2 r_{H}^{2}}\right)^{2}-\frac{a^{2} q^{2}}{2 L^{2} r_{H}^{2}}}{1-a^{2}\left(\frac{1}{L^{2}}+\frac{1}{r_{H}^{2}}\right)} .
$$

Working again in a non-rotating frame at infinity, the quantities which enter a thermodynamic description 
of the solutions, as expressed in terms of $r_{H}, a$ and $q$, read:

$$
\begin{aligned}
& M=\frac{\pi}{8} \frac{r_{H}^{2}}{\left(1-a^{2}\left(\frac{1}{L^{2}}+\frac{1}{r_{H}^{2}}\right)\right)}\left[\left(3+\frac{a^{2}}{L^{2}}\right)\left(1+\frac{r_{H}^{2}}{L^{2}}\right)+\frac{q}{r_{H}^{4}}\left(q\left(3-\frac{a^{4}}{L^{4}}-\frac{2 a^{2}}{L^{2}}\right)+2 a^{2}\left(3+\frac{4 r_{H}^{2}}{L^{2}}\right)\right)\right], \\
& Q=\frac{\sqrt{3} \pi}{2} q, \quad \Phi_{H}=\frac{\sqrt{3} q}{2 r_{H}^{2}} \frac{1-\frac{a^{2}}{r_{H}^{2}}\left(1+\frac{r_{H}^{2}}{L^{2}}\right)}{1+\frac{a^{2} q}{r_{H}^{4}}}, \quad \Omega_{H}=\frac{a}{L^{2}} \frac{1+\frac{L^{2}\left(q+r_{H}^{2}\right)}{r_{H}^{4}}}{1+\frac{a^{2} q}{r_{H}^{4}}}, \\
& J=\frac{\pi a}{4}\left[\frac{\frac{r_{H}^{4}}{L^{2}}+\frac{\left(q+r_{H}^{2}\right)^{2}}{r_{H}^{2}}-\frac{a^{2} q^{2}}{L^{2} r_{H}^{2}}}{1-a^{2}\left(\frac{1}{L^{2}}+\frac{1}{r_{H}^{2}}\right)}-q\right], \quad A_{H}=\frac{2 \pi^{2} r_{H}^{3}\left(1+\frac{a^{2} q}{r_{H}^{4}}\right)}{\sqrt{1-a^{2}\left(\frac{1}{L^{2}}+\frac{1}{r_{H}^{2}}\right)}}, \\
& T_{H}=\frac{1}{2 \pi r_{H}} \frac{\left(1+\frac{a^{2} q}{r_{H}^{4}}\right)^{-1}}{\sqrt{1-a^{2}\left(\frac{1}{L^{2}}+\frac{1}{r_{H}^{2}}\right)}}\left[\left(1-\frac{2 a^{2}+q}{r_{H}^{2}}\right)\left(1+\frac{q}{r_{H}^{2}}\right)-\frac{a^{2}\left(a^{2} q^{2}+2 r_{H}^{4}\right)}{L^{4} r_{H}^{4}}+\frac{2\left(r_{H}^{6}+a^{2}\left(q^{2}-2 r_{H}^{4}\right)\right.}{L^{2} r_{H}^{4}}\right] .
\end{aligned}
$$

The corresponding expressions for mass and angular momentum of the horizon are

$$
\begin{aligned}
& M_{H}=\frac{\pi}{4} \frac{a^{2} q+r_{H}^{4}}{L^{2}} \frac{2+\frac{a^{2} q}{r_{H}^{4}}\left(1+\left(1+\frac{L^{2}}{r_{H}^{2}}\right)\left(1+\frac{q}{r_{H}^{2}}\right)\right)+\frac{L^{2}}{r_{H}^{2}}\left(1-\frac{q^{2}}{r_{H}^{4}}\right)}{1-a^{2}\left(\frac{1}{L^{2}}+\frac{1}{r_{H}^{2}}\right)}, \\
& J_{H}=-\frac{\pi}{8} \frac{a\left(a^{2} q+r_{H}^{4}\right)}{L^{2}} \frac{\left(1+\frac{L^{2}}{r_{H}^{2}}\right)\left(2+\frac{2 a^{2} q^{2}}{r_{H}^{6}}+\frac{L^{2} q}{r_{H}^{4}}\left(2-\frac{q}{r_{H}^{2}}\right)\right)}{1-a^{2}\left(\frac{1}{L^{2}}+\frac{1}{r_{H}^{2}}\right)} .
\end{aligned}
$$

The parameters $\left(r_{H}, a, q\right)$ are subject to the condition

$$
\left(1-\frac{2 a^{2}+q}{r_{H}^{2}}\right)\left(1+\frac{q}{r_{H}^{2}}\right)-\frac{a^{2}\left(a^{2} q^{2}+2 r_{H}^{4}\right)}{L^{4} r_{H}^{4}}+\frac{2\left(r_{H}^{6}+a^{2}\left(q^{2}-2 r_{H}^{4}\right)\right.}{L^{2} r_{H}^{4}} \geq 0 .
$$

If the inequality in the equation above is saturated, the horizon is degenerate and we get an extremal $\mathrm{BH}$. With $q=0$, the CLP solutions reduce to MP-AdS spinning BHs with equal angular momenta [5]. Another limit of interest corresponds to $a=0$, in which case one recovers the RN-AdS BHs.

\section{A.2 Extremal limit and the critical solutions}

In discussing the $T_{H}=0$ limit of these solutions, it is convenient to reparametrize the constants $a, r_{H}$ as

$$
a=L x, \quad r_{H}=\frac{L x y}{\sqrt{1-x^{2}}},
$$

with $0 \leq x<1,1 \leq y<\infty$. Then the conditions $T_{H}=0$ is written as

$$
q=q_{ \pm}=\frac{L^{2} x^{2}}{\left(1-x^{2}\right)^{2}}\left(-1 \pm\left(y^{2}-1\right) \sqrt{1+2 y^{2} x^{2}}\right)
$$

which reveals the existence of two branches of extremal solutions, in terms of the parameters $(x, y)$. In particular, BHs with $T_{H}=0$ can be found for any value of $(Q, J)$.

The extremal BHs possess an interesting limit with a zero event horizon area, corresponding to the $\lambda=1$ critical solution discussed above. This limit is approached for

$$
y=\frac{r_{H}}{a} \sqrt{1-\frac{a^{2}}{L^{2}}} \rightarrow 1,
$$

on both branches of solutions. Interesting enough, the \pm global charges are the same as $y \rightarrow 1$ :

$$
M_{ \pm} \rightarrow \frac{L^{2} \pi}{8} \frac{x^{2}\left(x^{4}-3 x^{2}+6\right)}{\left(1-x^{2}\right)^{3}}, \quad J_{ \pm} \rightarrow \frac{L^{3} \pi}{4} \frac{x^{3}}{\left(1-x^{2}\right)^{3}}, \quad Q_{ \pm} \rightarrow-\frac{L^{2} \pi}{2} \frac{x^{2}}{\left(1-x^{2}\right)^{2}}<0
$$


while the \pm expressions of electrostatic potential and horizon angular velocity are different

$$
\Phi_{H \pm} \rightarrow-\frac{\sqrt{3}}{2\left(2 \pm \sqrt{1+2 x^{2}}\right)}, \quad \Omega_{H \pm} \rightarrow \frac{1}{L} \frac{1+x^{2} \pm \sqrt{1+2 x^{2}}}{x\left(2 \pm \sqrt{1+2 x^{2}}\right)} .
$$

This shows the existence of a discontinuity in both $\Phi_{H}$ and $\Omega_{H}$ as the limit $y \rightarrow 1$ is approached, with different limiting values for these quantities on each branch (although the global charges are equal).

The solution with $y=1$ has an interesting closed-form expression. After replacing A.6, A.8 in A.1) one finds

$$
\begin{aligned}
& F_{0}(r)=\frac{r^{2}\left(1-x^{2}\right)}{L^{2}} \frac{\left(r^{2}\left(1-x^{2}\right)+L^{2}\left(1+x^{2}\right)\right)\left(r^{2}\left(1-x^{2}\right)-L^{2} x^{2}\right)}{r^{4}\left(1-x^{2}\right)^{3}+r^{2} L^{2} x^{2}\left(1-x^{2}\right)^{2}+L^{4} x^{4}}, \\
& F_{1}(r)=\frac{L^{2} r^{4}\left(1-x^{2}\right)^{3}}{\left(r^{2}\left(1-x^{2}\right)+L^{2}\left(1+x^{2}\right)\right)\left(r^{2}\left(1-x^{2}\right)-L^{2} x^{2}\right)^{2}}, \quad F_{2}(r)=r^{2}, \\
& F_{3}(r)=\frac{r^{6}\left(1-x^{2}\right)^{4}+r^{2} L^{4} x^{6}\left(1-x^{2}\right)-L^{6} x^{6}}{r^{4}\left(1-x^{2}\right)^{4}}, \quad W(r)=\frac{L^{3} x^{3}}{r^{4}\left(1-x^{2}\right)^{3}+r^{2} L^{2} x^{2}\left(1-x^{2}\right)^{2}+L^{4} x^{4}}, \\
& a_{\varphi}(r)=\frac{\sqrt{3} L^{3}}{2 r^{2}} \frac{x^{3}}{\left(1-x^{2}\right)^{2}}, \quad a_{0}(r)=-\frac{\sqrt{3} L^{2}}{2 r^{2}} \frac{x^{2}}{\left(1-x^{2}\right)^{2}} .
\end{aligned}
$$

A direct inspection shows that this describes a BH spacetime, with standard AdS asymptotics. The event horizon is located at

$$
r=r_{H}=\frac{L x}{\sqrt{1-x^{2}}} \geq 0
$$

Despite possessing a zero horizon area, this configuration shows no (obvious) signs of a pathological behavior. For example, both the Ricci and Kretschmann scalar are finite on and outside the horizon.

Its near horizon expansion, $r \rightarrow r_{H}$ reads

$$
\begin{aligned}
& F_{0}(r)=\frac{2}{L} \frac{\sqrt{1-x^{2}}\left(1+2 x^{2}\right)}{x\left(3-2 x^{2}\right)}\left(r-r_{H}\right)+\ldots, \quad \frac{1}{F_{1}(r)}=\frac{4}{L^{2}} \frac{1+2 x^{2}}{x^{2}}\left(r-r_{H}\right)^{2}+\ldots, \\
& F_{2}(r)=r_{H}^{2}+\ldots, \quad F_{3}(r)=2 L \frac{x\left(3-2 x^{2}\right)}{\left(1-x^{2}\right)^{(3 / 2)}}\left(r-r_{H}\right), \\
& a_{\varphi}(r)=\frac{\sqrt{3} L}{2} \frac{x}{1-x^{2}}+\ldots, \quad a_{0}(r)=-\frac{\sqrt{3}}{2} \frac{1}{1-x^{2}}+\ldots, \quad w(r)=-\frac{1}{L} \frac{1}{x\left(3-2 x^{2}\right)}+\ldots,
\end{aligned}
$$

which, to leading order, describes an $\mathrm{AdS}_{3} \times S^{2}$ geometry. Other properties of this special solution have been already reported in the main text.

\section{References}

[1] S. W. Hawking and G. F. R. Ellis, "The Large scale structure of space-time," Cambridge University Press, Cambridge, 1973

[2] E. Witten, Adv. Theor. Math. Phys. 2 (1998) 253 [arXiv:hep-th/9802150].

[3] J. M. Maldacena, Adv. Theor. Math. Phys. 2 (1998) 231 [Int. J. Theor. Phys. 38 (1999) 1113] [arXiv:hepth/9711200].

[4] R. C. Myers and M. J. Perry, Annals Phys. 172 (1986) 304.

[5] S. W. Hawking, C. J. Hunter and M. M. Taylor-Robinson, Phys. Rev. D 59 (1999) 064005 [arXiv:hep-th/9811056].

[6] J. Kunz, F. Navarro-Lérida and E. Radu, Phys. Lett. B 649 (2007) 463 [gr-qc/0702086].

[7] M. Cvetič, H. Lü and C. N. Pope, Phys. Lett. B 598 (2004) 273 [arXiv:hep-th/0406196]. 
[8] J. B. Gutowski and H. S. Reall, JHEP 0402 (2004) 006 [hep-th/0401042].

[9] Z. W. Chong, M. Cvetič, H. Lü and C. N. Pope, Phys. Rev. Lett. 95 (2005) 161301 [arXiv:hep-th/0506029];

Z. W. Chong, M. Cvetič, H. Lü and C. N. Pope, Phys. Lett. B 644 (2007) 192 [arXiv:hep-th/0606213];

Z. W. Chong, M. Cvetič, H. Lü and C. N. Pope, Phys. Rev. D 72 (2005) 041901 [arXiv:hep-th/0505112];

M. Cvetič, H. Lü and C. N. Pope, Phys. Rev. D 70 (2004) 081502 [arXiv:hep-th/0407058];

[10] J. L. Blázquez-Salcedo, J. Kunz, F. Navarro-Lérida and E. Radu, Phys. Rev. Lett. 112 (2014) 011101 [arXiv:1308.0548 [gr-qc]].

[11] J. L. Blázquez-Salcedo, J. Kunz, F. Navarro-Lérida and E. Radu, Phys. Rev. D 92 (2015), 044025 [arXiv:1506.07802 [gr-qc]].

[12] J. L. Blázquez-Salcedo, J. Kunz, F. Navarro-Lérida and E. Radu, arXiv:1602.00822 [gr-qc].

[13] P. Figueras and S. Tunyasuvunakool, JHEP 1503 (2015) 149 [JHEP 1503 (2015) 149] [arXiv:1412.5680 [hep-th]].

[14] Y. Brihaye, B. Kleihaus, J. Kunz and E. Radu, JHEP 1011 (2010) 098 [arXiv:1010.0860 [hep-th]].

[15] O. J. C. Dias, G. T. Horowitz and J. E. Santos, JHEP 1107 (2011) 115 [arXiv:1105.4167 [hep-th]].

[16] S. Stotyn, M. Park, P. McGrath and R. B. Mann, Phys. Rev. D 85, 044036 (2012) [arXiv:1110.2223 [hep-th]].

[17] S. Stotyn, C. D. Leonard, M. Oltean, L. J. Henderson and R. B. Mann, Phys. Rev. D 89 (2014) 044017 [arXiv:1307.8159 [hep-th]].

[18] J. Kunz, F. Navarro-Lérida and J. Viebahn, Phys. Lett. B 639 (2006) 362 [arXiv:hep-th/0605075].

[19] J. Kunz and F. Navarro-Lérida, Phys. Lett. B 643 (2006) 55 [hep-th/0610036].

[20] Y. Brihaye, C. Herdeiro and E. Radu, Phys. Lett. B 739, 1 (2014) [arXiv:1408.5581 [gr-qc]].

[21] Y. Brihaye, C. Herdeiro and E. Radu, Phys. Lett. B 760, 279 (2016) [arXiv:1605.08901 [gr-qc]].

[22] A. N. Aliev, Phys. Rev. D 74 (2006) 024011

[23] A. N. Aliev and D. K. Ciftci, Phys. Rev. D 79 (2009) 044004

[24] M. Allahverdizadeh, J. Kunz and F. Navarro-Lérida, Phys. Rev. D 82 (2010) 024030 [arXiv:1004.5050 [gr-qc]].

[25] M. Allahverdizadeh, J. Kunz and F. Navarro-Lérida, Phys. Rev. D 82 (2010) 064034 [arXiv:1007.4250 [gr-qc]].

[26] H. K. Kunduri and J. Lucietti, JHEP 0712 (2007) 015 [arXiv:0708.3695 [hep-th]].

[27] A. Sen, JHEP 0509, 038 (2005) [hep-th/0506177].

[28] D. Astefanesei, K. Goldstein, R. P. Jena, A. Sen and S. P. Trivedi, JHEP 0610, 058 (2006) [hep-th/0606244].

[29] K. Goldstein and R. P. Jena, JHEP 0711, 049 (2007) [arXiv:hep-th/0701221].

[30] N. V. Suryanarayana and M. C. Wapler, Class. Quant. Grav. 24, 5047 (2007) [arXiv:0704.0955 [hep-th]].

[31] R. M. Wald, Phys. Rev. D 48, 3427 (1993) [gr-qc/9307038].

[32] J. Lee and R. M. Wald, J. Math. Phys. 31, 725 (1990).

[33] M. Rogatko, Phys. Rev. D 75, 024008 (2007) [hep-th/0611260].

[34] A. Ashtekar and A. Magnon, Class. Quant. Grav. 1 (1984) L39;

A. Ashtekar and S. Das, Class. Quant. Grav. 17, L17 (2000) [hep-th/9911230].

[35] V. Balasubramanian and P. Kraus, Commun. Math. Phys. 208 (1999) 413.

[36] D. Kastor, S. Ray and J. Traschen, Class. Quant. Grav. 26, 195011 (2009) [arXiv:0904.2765 [hep-th]].

[37] M. Cvetič, G. W. Gibbons, D. Kubiznak and C. N. Pope, Phys. Rev. D 84, 024037 (2011) [arXiv:1012.2888 [hep-th]].

[38] U. Ascher, J. Christiansen, R. D. Russell, Mathematics of Computation 33 (1979) 659; ACM Transactions 7 (1981) 209.

[39] M. Cvetič, G. W. Gibbons, H. Lü and C. N. Pope, "Rotating black holes in gauged supergravities: Thermodynamics, supersymmetric limits, topological solitons and time machines", hep-th/0504080.

[40] S. W. Hawking and D. N. Page, Commun. Math. Phys. 87 (1983) 577.

[41] A. N. Aliev and V. P. Frolov, Phys. Rev. D 69 (2004) 084022 [hep-th/0401095]. 
[42] A. N. Aliev, Class. Quant. Grav. 24 (2007) 4669 [hep-th/0611205];

A. N. Aliev, Phys. Rev. D 75 (2007) 084041 [hep-th/0702129].

[43] J. L. Blázquez-Salcedo, "Radially excited AdS 5 black holes in Einstein-Maxwell-Chern-Simons theory", to appear.

[44] M. Mir and R. B. Mann, Phys. Rev. D 95 (2017) 024005

[45] H. K. Kunduri and J. Lucietti, Nucl. Phys. B 724, 343 (2005) [hep-th/0504158].

[46] O. Madden and S. F. Ross, Class. Quant. Grav. 22 (2005) 515 [hep-th/0409188].

[47] P. Davis, H. K. Kunduri and J. Lucietti, Phys. Lett. B 628 (2005) 275 [hep-th/0508169]. 